3

4

\title{
Mechanisms of genetic instability in a single S-phase following whole genome doubling
}

Simon Gemble ${ }^{1,7}$, Sara Vanessa Bernhard ${ }^{2}$, Nishit Srivastava ${ }^{3}$, René Wardenaar ${ }^{4}$ Maddalena Nano ${ }^{1}$, Anne-Sophie Macé ${ }^{5}$, Andréa E. Tijhuis ${ }^{4}$, Kristina Keuper ${ }^{2}$, Diana C.J. Spierings ${ }^{4}$, Helfrid Hochegger ${ }^{6}$, Matthieu Piel ${ }^{3}$, Floris Foijer ${ }^{4}$, Zuzana Storchová2 $^{2}$ and Renata Basto ${ }^{1,7}$.

1- Institut Curie, PSL Research University, CNRS, UMR144, Biology of Centrosomes and Genetic Instability Laboratory, Paris, France.

2- Department of Molecular Genetics, TU Kaiserslautern, Kaiserslautern, Germany.

3- Institut Curie and Institut Pierre Gilles de Gennes, PSL Research University, CNRS, UMR 144, Systems Biology of Cell Polarity and Cell Division, Paris, France.

4- European Research Institute for the Biology of Ageing, University of Groningen, University Medical Center Groningen, Groningen, The Netherlands.

5- Cell and Tissue Imaging Facility (PICT-IBiSA), Institut Curie, PSL Research University, Centre National de la Recherche Scientifique, Paris, France.

6- Genome Damage and Stability Centre, School of Life Sciences, University of Sussex, Brighton, UK.

7- Corresponding authors- simon.gemble@curie.fr and renata.basto@curie.fr 


\section{ABSTRACT}

32

Doubling of the full chromosome content -whole genome duplications (WGDs)- is frequently found in human cancers and is responsible for the rapid evolution of genetically unstable karyotypes ${ }^{1-3}$. It has previously been established that WGDs fuel chromosome instability due to abnormal mitosis owing to the presence of extra centrosomes and extra chromosomes ${ }^{4-8}$. Tolerance to ploidy changes has been identified in different model organisms and cell types ${ }^{5,6,9-12}$, revealing long term cellular adaptations that accommodate ploidy increase. Importantly, however, the immediate consequences of WGDs as cells become tetraploid are not known. It also remains unknown whether WGD triggers other events leading to genetic instability (GIN), independently of mitosis. In this study, we induced tetraploidy in diploid genetically stable RPE-1 cells and monitored the first interphase. We found that newly born tetraploids undergo high rates of DNA damage during DNA replication. Using DNA combing and single cell sequencing, we show that replication forks are unstable, perturbing DNA replication dynamics and generating under- and over-replicated regions at the end of S-phase. Mechanistically, we found that these defects result from lack of protein mass scaling up at the G1/S transition, which impairs the fidelity of DNA replication. This work shows that within a single interphase, unscheduled tetraploid cells can accumulate highly abnormal karyotypes. These findings provide an explanation for the GIN landscape that favors tumorigenesis after tetraploidization. 


\section{MAIN}

55 Diploid and stable karyotypes are associated with health and fitness in animals. In 56 contrast, whole genome duplications (WGDs) are linked to genetic instability (GIN) and cancer ${ }^{1,2,13}$. WGDs promote chromosomal instability (CIN) over time due to abnormal mitosis, contributing to the evolution of aneuploid karyotypes ${ }^{3,6,8,14-16}$. These represent cancer vulnerabilities with therapeutic potential ${ }^{17,18}$. Tolerance to ploidy changes has been identified in different model organisms and cell types ${ }^{5,6,9-12}$, revealing long-term

61 cellular adaptations that accommodate ploidy increase. Importantly, however, the

62 immediate consequences of unscheduled WGDs are not known and their contribution

63 to GIN remains to be identified. This is an essential question because single WGD 64 events such as cytokinesis failure can promote tumorigenesis ${ }^{19}$. Identifying the initial 65 defects derived from WGD and the underlying mechanisms establishing GIN in 66 tetraploid cells is thus an important step, which requires understanding failure in 67 maintaining genetic stability. This type of study has the potential to unravel the origins 68 of GIN in one single cell cycle after WGDs. 
High levels of DNA damage are generated in the first interphase following unscheduled Whole Genome Duplication (WGD)

To identify the immediate consequences of WGDs, we induced tetraploidization in the human diploid and genetically stable RPE-1 immortalized cell line, which contains an almost near-diploid chromosome content. Since WGDs can have different origins ${ }^{15,20}$, we developed several approaches to induce tetraploidization through either cytokinesis failure (CF), endoreplication (EnR) or mitotic slippage (MS) (Fig. 1A and methods). While the large majority of cells resulting from CFs contained two nuclei, EnR or MS generated mainly mononucleated tetraploid cells (Fig. 1A-B and Extended data Fig. 1A-I). Importantly, taking together parameters such as cell size, nuclei number and size, and centrosome number, we were able to distinguish diploids from tetraploids in all the strategies used (Fig. 1B and Extended data Fig. 1A-I). For each strategy, a mix of diploid and tetraploid cells was obtained, allowing a comparison of an internal diploid control and the tetraploid population (Fig. $1 \mathrm{C}-\mathrm{H}$, and Extended data Fig. 1A-I). Importantly, from all the conditions used to induce WGDs, the large majority of tetraploid cells continued to cycle and enter and exit the first S-phase (see below). Thus, we have generated the conditions required to study the initial and immediate consequences of tetraploidy within the first cell cycle.

Using an early marker of DNA double strand breaks - $\gamma \mathrm{H} 2 \mathrm{AX}-$, we characterized levels of DNA damage in the first interphase following tetraploidization and found high levels in tetraploid cells. Moreover, this was independent of the way tetraploid cells were generated. In contrast, diploid cells treated in the same conditions or untreated diploids showed low levels of DNA damage (Fig. 1C-H, and Extended data Fig. 1A-I, see Extended data Fig. 2A-C for additional methods of generating tetraploidy). While most of the diploid cells exhibited a low number of $\gamma \mathrm{H} 2 \mathrm{AX}$ foci, the percentage of tetraploid cells with more than 10 foci was high (Fig. 1C-H). We found a correlation between the number of $\gamma \mathrm{H} 2 \mathrm{AX}$ foci and the fluorescence intensity levels of this marker (Extended data Fig. 1J). For simplicity, we will include the information of the number of $\gamma \mathrm{H} 2 \mathrm{AX}$ foci per interphase nuclei throughout this study. Since MS generated the highest frequency of tetraploid cells (Extended data Fig. 1A), we chose to present data derived from MS throughout this study. To confirm some of our results, we also used CF or EnR and this will be mentioned in the figure legends and in the methods. 
Since a tetraploid nucleus contains twice the amount of DNA than a diploid nucleus, we excluded by normalization with nuclear area or nuclear fluorescence intensity, an increase of $\gamma \mathrm{H} 2 \mathrm{AX}$ foci due to increased nuclear size (Extended data Fig. $1 \mathrm{~K}-\mathrm{L})$. Additionally, we confirmed that the high levels of DNA damage found in the first interphase after tetraploidization were not specific to RPE-1 cells, as the diploid BJ fibroblast cell line as well as the pseudo diploid human colon carcinoma HCT116 cell line also displayed high levels of DNA damage upon WGD (Extended data Fig. 2D-E).

We next compared the levels of DNA damage detected in tetraploid cells with the levels of DNA damage in diploid cells generated by replication stress (RS). RS is the slowing or stalling of replication forks, which can be induced by high doses of Aphidicolin (APH) or Hydroxyurea (HU), among other challenges ${ }^{21,22}$. Interestingly, $\mathrm{APH}$ or $\mathrm{HU}$ generated comparable levels of DNA damage in diploid cells, when compared to untreated tetraploid cells (Extended data Fig. 1M).

Collectively, our results show that a transition from a diploid to tetraploid status after unscheduled WGD, is accompanied by high levels of DNA damage within the first cell cycle.

DNA damage and genetic instability in tetraploid cells is generated during Sphase in a DNA replication-dependent manner

We then determined the cell cycle stage in which the DNA damage occurs. We followed cell cycle progression right after WGD using the fluorescence ubiquitination cell cycle indicator (FUCCl) system to map the timing of cell cycle progression in tetraploid cells, allowing us to then monitored the number of $\gamma \mathrm{H} 2 \mathrm{AX}$ foci during the first

125 G1 and the first S-phase (Fig. 2A-B and Extended data Fig. 3A). During G1, the number 126 of $\gamma \mathrm{H} 2 \mathrm{AX}$ foci was quite low and comparable to controls. As cells enter S-phase $127(t=10 \mathrm{hrs})$, a slight increase in the number of foci in tetraploid nuclei could be observed, which increased substantially at the end of S-phase ( $\mathrm{t}=16 \mathrm{hrs}$ ) (Fig. 2A-B and Extended data Fig. 3A). These results were further confirmed by time lapse imaging using tetraploid RPE-1 cells tagged with H2B-GFP to visualize DNA and 53BP1-RFP, which is a double strand break repair factor ${ }^{23}$ (Extended data Fig. 3B-C and Extended data movies 1-2). To confirm that DNA damage in tetraploid cells was induced during S-

133 phase, we blocked cells at the G1/S transition using high doses of either CDK4/6 or 134 CDK2 inhibitors for 16 hrs (methods). We chose the 16hrs time period because it 
136 CDKs inhibitors were washed out allowing cell cycle progression (Extended data Fig. 137 3D). G1-arrested tetraploid cells showed low levels of DNA damage, whereas cells 138 released in S-phase exhibited high levels of DNA damage (Extended data Fig. 3D-G). 139 Importantly, a certain proportion of $\gamma \mathrm{H} 2 \mathrm{AX}$ foci of S-phase tetraploid cells partially co140 localized with markers of active DNA replication sites visualized by Proliferating Cell 141 Nuclear Antigen (PCNA) and EdU incorporation (Extended data Fig. 3H).

142 To better characterize DNA damage in tetraploid cells during their first 143 interphase, we used other markers of the DNA damage signaling and repair pathways.

144 We found that the number of KU80 and XRCC1 foci, two proteins involved in Non145 Homologous End Joining (NHEJ) ${ }^{24}$ remained low (Extended data Fig. 4A-B). In 146 contrast, the number of RAD51 foci, a protein involved in homologous recombination $147(\mathrm{HR})$, was increased and co-localized with $\gamma \mathrm{H} 2 \mathrm{AX}$ in a fraction of tetraploid cells 148 (Extended data Fig. 4C-D). This was also the case for Replication protein A (RPA) and 149 FANCD2 foci, two markers of RS which also co-localized with $\gamma \mathrm{H} 2 \mathrm{AX}$ foci in tetraploid cells (Extended data Fig. 4E-G). Together, these results demonstrate that tetraploid cells experience high levels of DNA damage during S-phase progression, which are recognized by bonafide DNA damage markers and by the HR repair pathway.

Based on these results, we hypothesized that DNA damage in tetraploid cells arises from errors occurring during DNA replication. To test this possibility, we arrested cells in $\mathrm{G} 1$ as described above (Extended data Fig. 3D). We then released them in the presence of very low doses of APH or PHA, a Cdc7 inhibitor. We used low doses of these compounds to inhibit DNA replication (detected by absence of EdU) without generating DNA damage (methods). These conditions resulted in the inhibition of DNA replication, albeit maintaining the biochemical activity typical of the S-phase nucleus.

160 Strikingly, the levels of DNA damage in tetraploid cells were dramatically decreased 161 when DNA replication was inhibited (Fig. 2C-D and Extended data Fig. 4H-J). 162 Importantly, in the few tetraploid cells that escaped DNA replication inhibition revealed by high EdU incorporation - a high number of $\gamma \mathrm{H} 2 \mathrm{AX}$ foci were noticed

164 (Extended data Fig. 4K-L), confirming the correlation between DNA replication and DNA damage in tetraploid cells.

To characterize DNA replication, we established RPE-1 cell lines stably expressing PCNA chromobodies. We showed that expression of PCNA chromobodies does not affect cell cycle progression in RPE-1 cells, confirming its suitability to follow DNA replication by live imaging (Extended data Fig. 4M). Using this cell line, we 
170 performed quantitative 4D live imaging of endogenous DNA replication in diploid and 171 tetraploid cells (methods). Surprisingly, the comparison between the total number of 172 PCNA foci during S-phase in diploid and tetraploid RPE-1 cells, revealed a lack of 173 scaling up with DNA content (Fig. 2E-G), also observed by quantifying the number of 174 EdU foci (Extended data Fig. 4N). This result suggests that fewer replication sites were 175 activated in tetraploid S-phase cells, when compared with diploid cells. The volume of 176 PCNA foci was also lower in tetraploid cells (Fig. 2H). Moreover, timelapse analysis of 177 PCNA dynamics revealed additional differences. As diploid cells enter S-phase, an exponential increase in the number of active replication sites was noticed, which was maintained before undergoing a steep, almost abrupt decreased (Fig. 2E-I, Extended data Fig. 5A and Extended data movie 3). In contrast, in tetraploid cells the increase in the number of active sites was more gradual, and the signals associated with DNA 182 lingered for extended periods of time. Furthermore, the dissociation of PCNA from the 183 DNA in tetraploid cells occurred much later and also in a progressive manner (Fig. 2E184 I, Extended data Fig. 5A and Extended data movie 4). In line with this, by analyzing PCNA patterns as a readout of early and late S-phase ${ }^{25}$, we showed that tetraploid cells spent more time in early S-phase compared to late S-phase (Extended data Fig. 40). Surprisingly, even if S-phase was longer in tetraploid cells when compared to diploid cells (Extended data Fig. 4P), this was not sufficient to scale the number of active replication sites with DNA content. These results suggest that DNA replication in tetraploid cells is impaired due to both a lack of scaling up in the number of active replication sites and to a delayed DNA replication timing.

To ascertain if these defects impacted replication fork progression, we performed DNA combing, which allows the visualization of replication origins in single DNA fibers ${ }^{26}$. We failed to obtain fibers of the required quality in RPE-1 cells despite several attempts. To overcome this problem, we performed DNA combing in HCT116

196 cells since they also showed high levels of DNA damage within the first interphase 197 (Extended data Fig. 2E). Inter-origin distances were not affected in tetraploid cells, 198 however, and surprisingly, median fork speed was increased in tetraploid cells (Fig. 2J and Extended data Fig. 4Q). Further, a high increase in the percentage of unstable

200 forks was also detected (Fig. 2J). These results show that the replication dynamics is perturbed in tetraploid cells when compared to diploid cells. 
tetraploidy was associated with abnormal karyotypes. We FACS sorted tetraploid from diploid cells (see below, Fig. 3C) in G1 and at the G2/M transition to perform single cell DNA sequencing (ssDNAseq) (methods). Normalization of the under and over replicating regions in $\mathrm{G} 1$ and $\mathrm{G} 2 / \mathrm{M}$ diploid cells revealed already whole chromosome deviations in a certain number of cells. When present, they span along almost all chromosomes of a given cell (Extended data Fig. 5B). In G1 tetraploid cells, over replicated regions $(5 n)$ could also be identified, but these were restricted to a few chromosomes and might be explained by a caveat of the method (cells have initiated S-phase but were still selected as G1 by the FACS profile). Striking, however in G2/M

213 tetraploid cells over duplicating chromosomes $(>10)$ could be identified in addition to 214 frequent over and under replicated regions (9n, 7n and 4n) (Fig. 2K). In agreement with this variability and the extent of copy number deviations, both aneuploidy score and heterogeneity score were increased in G2/M tetraploid cells when compared to G2/M diploid cells (Aneuploidy score: 0.275 vs 0.102 ; Heterogeneity score 0.319 vs 0.158 respectively) (methods).

Together, our results show that unscheduled tetraploid cells cannot sustain normal DNA replication as they fail to scale proportionally the number of active replication sites and replication timing. Defects in S-phase result in the generation of highly aberrant karyotypes, demonstrating a causal relationship between tetraploidization and GIN within a single S-phase.

\section{Lack of G1 lengthening in tetraploid cells leads to unprepared S-phase}

The massive GIN described above, together with abnormal DNA replication dynamics, suggested that newly born tetraploid cells undergo the first S-phase in a non-optimal manner. We reasoned that cells might enter S-phase without the required protein levels to replicate a tetraploid genome. In principle, doubling the whole set of chromosomes should lead to an overall doubling of transcripts and protein translation, so that tetraploid cells should scale up by a factor of 2 . To determine if cell mass was

232 increased in tetraploid cells at the G1-S transition, we combined quantitative phase imaging with the cell cycle sensor $\mathrm{FUCCl}$, which allows recording of mass measure

234 trajectories through the cell cycle at the single cell level ${ }^{27}$. We found that the proportion 235 of mass added during G1 was lower in tetraploid cells compared to diploid cells (Fig. $2363 \mathrm{~A}-\mathrm{B})$. These results establish that newly born tetraploid cells are not able to scale 237 together protein and DNA content during the first G1 upon WGD. We next tested the 
238 total levels of key S-phase components. To do so, we used a recently developed 239 protocol that enables to sort and isolate tetraploid from diploid cells based on $\mathrm{FUCCl}$ 240 and DNA content from a common population (Fig. 3C and Extended data Fig. 6A, 241 methods). Protein extracts from the two cell populations at the G1/S transition were 242 then probed by western blot. The same number of cells was loaded for diploid and 243 tetraploid conditions. Normalization of the chromatin associated H2B variant and the 244 cytoskeleton component Actin showed an increase in these two protein levels consistent with DNA doubling (Fig. 3D-E and Extended data Fig. 6B). In stark contrast, essential S-phase DNA replication factors such as the origin recognition complex 1 (ORC1) involved in the recognition of replication origins ${ }^{28}$, the minichromosome maintenance 2 (MCM2) helicase ${ }^{29}$, CDC45, a member of the active helicase complex ${ }^{30}$, and PCNA did not scale up in tetraploid cells (Fig. 3D-F). Combined with quantitative phase imaging data, these results suggest that tetraploid cells do not contain the required protein levels to sustain timely and successful DNA replication during S252 phase.

In normal proliferative cell cycles, the growth phase occurring during G1 phase prepares cells for DNA replication allowing the expression and accumulation of key Sphase regulators ${ }^{31,32}$. We reasoned that a short $\mathrm{G} 1$ duration could account for transition to S-phase in an unprepared manner, which is supported by the fact that tetraploid cells did not scale up protein content with DNA doubling (Fig. 3A-F). Indeed, time lapse analysis of tetraploid cells just after birth indicated only a slight increase in G1 duration, which did not scale with DNA content when compared to diploid cells (Fig. $3 G)$. Further, while we observed a significant correlation between cell mass and G1 duration in diploid cells, also described in other diploid conditions ${ }^{33}$, this correlation was absent in tetraploid cells suggesting that $\mathrm{G} 1$ duration is not dependent of cell mass in tetraploid cells (Fig. 3H).

We next tested if imposing G1 lengthening was translated by increased protein levels of S-phase factors and thus in principle enabled error-free DNA replication in 266 tetraploid cells. To test this model, we delayed S-phase entry using very low doses of CDK4/6 or CDK2 inhibitors. These conditions were different from the ones described above used to synchronize cells in G1. Indeed, while high doses of these inhibitors result in a biochemical arrest, low inhibitor doses result in $\mathrm{G} 1$ lengthening ${ }^{34,35}$. The different impact of high and low doses of CDK4/6 or CDK2 inhibitors could be noticed by differences in the expression levels of DNA replication factors. Indeed, after G1 
272 lengthening the levels of DNA replication factors scaled up with DNA content in

273 tetraploid cells, which was not the case when cells were arrested in G1 (Fig. 3I-K vs

274 3D-F). Consistent with these findings, after $\mathrm{G} 1$ lengthening the number and volume of 275 active replication sites in the subsequent S-phase visualized by monitoring PCNA or 276 EdU foci scaled up with DNA content in tetraploid cells (Extended data Fig. 6C-E). 277 Moreover, after G1 extension, PCNA dynamic behavior in tetraploid cells was 278 comparable to diploid cells (Fig. 3L and Extended data Fig. 6F and I and Extended 279 data movies 5-6). Even if the time spent in S-phase was not altered after G1 280 lengthening, we observed that increasing G1 duration restored the ratio between early 281 and late S-phase in tetraploid cells, suggesting that DNA replication timing was 282 reinstated (Extended data Fig. 6G-H). Strikingly, G1 lengthening was sufficient to 283 significantly reduce DNA damage in tetraploid S-phase cells (Fig. 3M and Extended 284 data Fig. 6J-L).

Altogether, our data show that tetraploid cells transition from G1 to S-phase prematurely without undergoing scaling of global protein mass, and so they enter in Sphase with insufficient amounts of DNA replication factors. This impacts the dynamics and fidelity of DNA replication, generating DNA damage. Importantly, extension of G1 is sufficient to increase the levels of key DNA replication factors, which results in a significant decrease in DNA damage in tetraploid cells.

G1 lengthening or increased E2F1 levels are sufficient to rescue GIN in tetraploid cells and in polyploid cells in vivo

From yeast to mammals, the transition from $\mathrm{G} 1$ to S-phase is negatively regulated by members of the retinoblastoma $(\mathrm{Rb})$ protein family, which sequesters the transcription factor E2F1 ${ }^{31,32,36-38}$. E2F1 targets several genes required for entry into S-phase and DNA replication factors. Since a short G1 does not prepare tetraploid cells for S-phase, we reasoned that increased levels of E2F1 might override the G1 lengthening defect. We thus expressed E2F1 in diploid cells (Extended data Fig. 7A), allowing to increase the expression of DNA replication proteins just before generating tetraploid cells. Importantly, this was sufficient to rescue the levels of DNA damage in tetraploid cells (Fig. 4A-B).

303 We recently characterized an in vivo model to study the consequences of 304 polyploidy in Drosophila neural stem cells also called neuroblasts (NBs) in the 305 developing brain ${ }^{39}$. These cells are normally diploid, but through repeated CF can 
generate highly polyploid NBs (here referred to as unscheduled polyploidy) much beyond the tetraploidization status (Fig. 4C-D and Extended data Fig. 7B). A key prediction of our findings is that polyploid NBs should also accumulate high levels of DNA damage in vivo. To test this prediction, we determined the levels of DNA damage in unscheduled polyploid NBs during interphase using antibodies against $\gamma \mathrm{H} 2 \mathrm{Av}$ to determine the $\gamma \mathrm{H} 2 \mathrm{Av}$ index (methods). We compared it with diploid NBs and the programmed polyploid cells from the Drosophila salivary gland, which are extremely large and accumulate more than 2000 chromosomes ${ }^{40}$. This represents a 250 - fold increase in DNA content, when compared with diploid Drosophila cells, which contain only 8 chromosomes. Interestingly, interphase polyploid NBs displayed high levels of DNA damage, which was not the case in diploid NBs or polyploid cells from the salivary gland (Fig. 4D-E and Extended data Fig. 7C). We next increased the levels of E2F1 and $\mathrm{Rb}$ by over-expressing (OE) in a tissue-specific manner using the UAS-Gal4 system. E2F1OE increases the expression of cell cycle regulators, while RbOE increases $\mathrm{G} 1$ lengthening 41,42 . Strikingly, this was sufficient to decrease substantially the levels of DNA damage in unscheduled polyploid NBs in vivo (Fig. 4F-G and Extended data Fig. 7D).

Taken together, these data show that in vivo unscheduled polyploidy is a source of DNA damage and GIN, which can be inhibited by increased E2F1 or Rb levels. These results put forward the idea that lack of cell cycle readjustment promotes GIN and the accumulation of highly complex karyotypes in cells that are not programmed to increase their DNA content (Fig. $4 \mathrm{H})$.

Here, we analyzed the initial defects following WGD and identified a very early window of high GIN that could promote acquisitions of multiple mutations making it possible to bypass cell cycle controls while promoting tetraploid cell survival. Our results are consistent with a model where tetraploid cells transit through the first cell cycle without preparing the duplication of increased DNA content (Fig. 4H). We found defective fork progression rates in tetraploid cells, which surprisingly seem to progress faster than in diploid cells. Although the increased rates remain to be explained, these may contribute to RS and GIN as recently shown upon PARP inhibition ${ }^{43}$ or in response to decreased levels of MCM proteins ${ }^{44}$. Strikingly, G1 extension or the increase in the expression levels of cell cycle proteins in vivo lowered considerably the high DNA damage levels of extreme polyploid cells such as the ones generated by 
340 repeated CF. These results highlight the importance of keeping constant scaling up

341 between DNA and protein content to ensure genetic stability and cell homeostasis.

342 The most surprising finding of this study is the lack of scaling up between DNA

343 and protein content immediately after tetraploidization. In physiological conditions,

344 such as during animal development, WGDs and polyploidization lead to an overall

345 scaling up of cell mass and DNA content to favor increase in secretion and metabolic

346 activity for example $20,45,46$. Our work shows that unscheduled tetraploid or polyploid

347 cells do not increase cell mass as expected. Why certain key cell cycle and DNA

348 replication factors fail to be expressed at levels that allow optimal DNA replication

349 remains to be explained. Importantly, however these results show that an immediate

350 consequence of unscheduled genome doubling is loss of genetic integrity within a

351 single S-phase. Interestingly, studies performed on stable tetraploid cells have shown

352 a remarkable scaling up between protein and DNA content after long term adaptation

35347,48 . It is tempting to propose that in non-physiological conditions, as the ones studied

354 here, newly born tetraploids do not "feel" the increase in DNA content and so, cannot

355 adapt $\mathrm{G} 1$ duration or protein content in order to replicate a $4 \mathrm{~N}$ genome. It will be

356 interesting to identify the molecular mechanisms that promote ploidy increase while

357 maintaining genetic stability and cell homeostasis. 


\section{Acknowledgments}

360 The authors acknowledge the Cell and Tissue Imaging platform (PICT-IBiSA), member

361 of the French National Research Infrastructure France-Biolmaging (ANR10-INBS-04)

362 and the Nikon Imaging center from Institut Curie for microscopy. We thank $L$.

363 Guyonnet, A. Chipont and C. Guerrin from the Cytometry platform of Institut Curie. We

364 thank V. Marthiens, S. Lambert, E. Schwob, J.S. Hoffmann, D. Fachinetti, M. Budzyk,

365 F. Edwards, O. Goundiam, G. Fantozzi, R. Salamé, A. Goupil and C. Chen for helpful 366 discussions and/or comments on the manuscript. This work was supported by 367 FOR2800/STO918-7 to Z.S, ERC CoG (ChromoNumber-LS3, ERC-2016-COG) for 368 R.B, Institut Curie and the CNRS. The Basto lab is a member of the Cell(n)Scale 369 Labex.

\section{Author contributions}

372 S.G. and R.B. conceived the project and wrote the manuscript. S.G. did most of the 373 experiments and data analysis presented here. M.N. did the initial observations of high 374 levels of DNA damage in Drosophila polyploid NBs. S.V.B., K.K. and Z.S. did the DNA 375 combing. R.W., A.E.T., D.C.J.S. and F.F. did the scSeq and BI analysis. A.S.M helped 376 with image quantifications and analysis. N.S. and M.P. performed the quantitative 377 phase imaging experiments and analysis and H.H. contributed with unpublished cell 378 lines. All authors read and comment on the manuscript. 
381 Cell culture, generation of cell lines and treatments:

382 Cell culture:

383 Cells were maintained at $37^{\circ} \mathrm{C}$ in a $5 \% \mathrm{CO}_{2}$ atmosphere. hTERT RPE- 1 cells (ATCC 384 Cat\# CRL-4000, RRID:CVCL_4388) and HEK293 cells (ATCC Cat\# CRL-1573, 385 RRID:CVCL_0045) were grown in Dulbecco's modified medium (DMEM) F12 (11320386033 from Gibco) containing 10\% fetal bovine serum (GE Healthcare), $100 \mathrm{U} / \mathrm{ml}$ 387 penicillin, $100 \mathrm{U} / \mathrm{ml}$ streptomycin (15140-122 from Gibco). BJ cells (ATCC Cat\# CRL388 4001, RRID:CVCL_6573) and HCT116 cells (ATCC Cat\# CCL-247, 389 RRID:CVCL_0291) were grown in Dulbecco's modified medium + GlutaMAX (61965390026 from Gibco) containing 10\% fetal bovine serum (GE Healthcare), $100 \mathrm{U} / \mathrm{ml}$ 391 penicillin, $100 \mathrm{U} / \mathrm{ml}$ streptomycin (15140-122 from Gibco).

All cells were routinely checked for mycoplasma infection.

Generation of RPE-1 PCNA ${ }^{\text {chromo }}$ stable cell line:

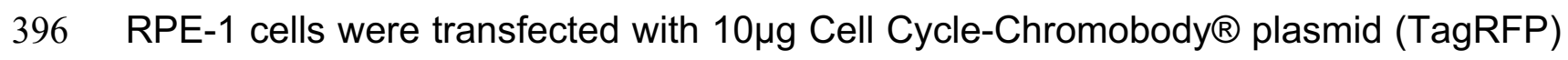
397 (From Chromotek, Planegg, Germany) using JET PRIME kit (Polyplus Transfection, 398 114-07) according to the manufacturer protocol. After 24 hours, $500 \mu \mathrm{g} / \mathrm{ml} \mathrm{G418}$ 399 (4727878001 from Sigma Aldrich) was added to the cell culture medium and then 400 clones expressing PCNA chromobodies were selected.

Generation of a RPE-1 FUCCI or RPE-1 CCNB1 ${ }^{A I D}$ FUCCI stable cell line:

To produce lentiviral particles, HEK293 cells were transfected with $4 \mu \mathrm{g}$ pBOB-EF1-

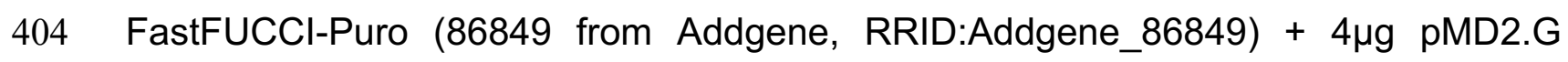

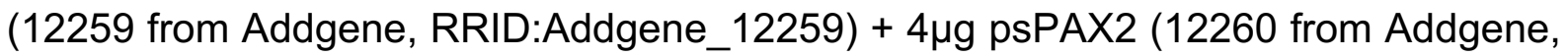
406 RRID:Addgene_12260) using FuGENE HD Transfection Reagent (E2311 from 407 Promega) in OptiMEM medium (51985034 from ThermoFisher). Cells were incubated 408 at $37^{\circ} \mathrm{C}$ in a $5 \% \mathrm{CO} 2$ atmosphere for 16 hours and then growth media were removed and replaced by $5 \mathrm{ml}$ fresh OptiMEM. The following day, viral particles were isolated 410 by filtering the medium containing the viral particles through a $0.45 \mu \mathrm{m}$ filter (16537 411 from Sartorius stedim biotech). Then, RPE-1 or RPE-1 CCNB1AID 49 cells were 412 incubated with viral particles in the presence of $8 \mu \mathrm{g} / \mathrm{ml}$ polybrene (sc-134220 from 413 Santa $\mathrm{Cruz}$ ) at $37^{\circ} \mathrm{C}$ in a $5 \% \mathrm{CO}_{2}$ atmosphere for 24 hours. RPE-1 GFP and RFP- 
414 positive cells were then collected using Sony SH800 FACS (BD FACSDiva Software 415 Version 8.0.1). RPE-1 or RPE-1 CCNB1AID clones expressing FUCCl were selected 416 and the cell lines were established from one single clone.

417 pBOB-EF1-FastFUCCI-Puro was a gift from Kevin Brindle \& Duncan Jodrell (Addgene 418 plasmid \# 86849 ; http://n2t.net/addgene:86849 ; RRID:Addgene_86849) ${ }^{50}$.

420 Generation of RPE-1 GFP-53BP1 RFP-H2B stable cell line:

421 This cell line was obtained as described below. Briefly, to produce lentiviral particles,

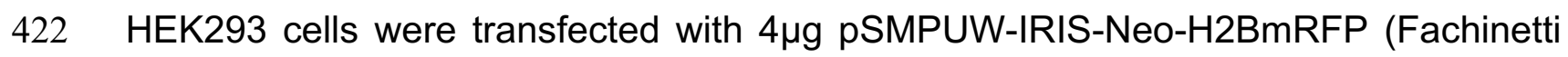

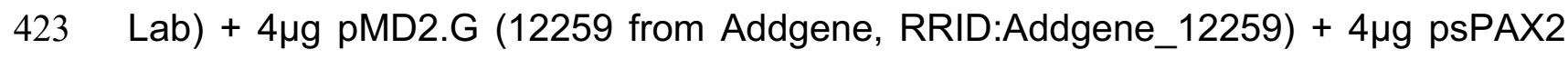
424 (12260 from Addgene, RRID:Addgene_12260). Then, RPE-1 cells were incubated with 425 viral particles and RPE-1 RFP-positive cells were collected using Sony SH800 FACS 426 (BD FACSDiva Software Version 8.0.1). RPE-1 clones expressing RFP-H2B were 427 selected, and the cell line was established from one single clone.

428 Then, new lentiviral particles were produced by transfecting HEK293 cells with $4 \mu \mathrm{g}$

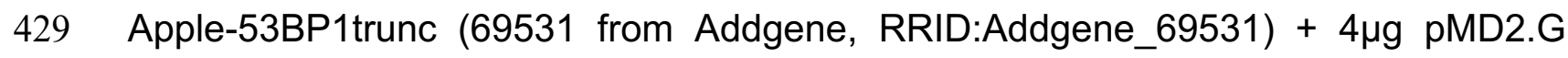

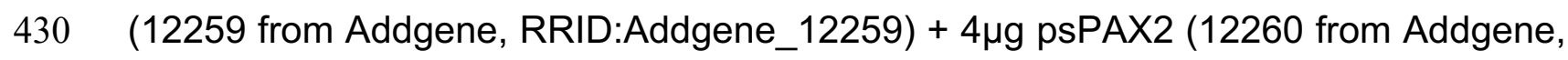
431 RRID:Addgene_12260). RPE-1 RFP-H2B cells were incubated with viral particles and 432 RPE-1 clones expressing both RFP-H2B and GFP-53BP1 were selected using flow 433 cytometry (FACS SH800 from Sony) and the cell line was established from one single 434 clone.

435 Apple-53BP1trunc was a gift from Ralph Weissleder (Addgene plasmid \# 69531 ; http://n2t.net/addgene:69531; RRID:Addgene_69531) ${ }^{51}$.

Generation of tetraploid cells:

439 Mitotic slippage using drugs: cells were incubated with DMSO (D8418 from Sigma

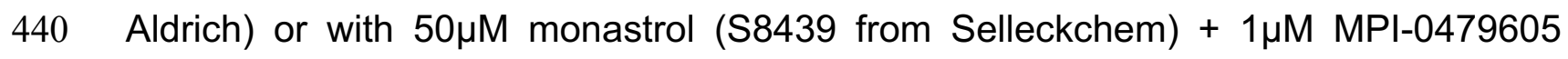
441 (S7488 from Selleckchem) for at least two hours. This approach was used in Fig. 1B442 D; Fig. 2A-I: Fig. 3L-M; Fig. 4A-B; Extended data Fig. 1A-C and I-M; Extended data 443 Fig. 2D-E; Extended data Fig.3B-E and G-H; Extended data Fig. 4A-H; K and N-P; 444 Extended data Fig. 5A; Extended data Fig. 6C-H and J-L; Extended data Fig. 7A.

445 Mitotic slippage using genetic tools: CCNB1 depletion in RPE CCNB1 ${ }^{\text {AID }}$ cells was 446 induced as described before ${ }^{49}$. Briefly, cells were treated with $2 \mu \mathrm{g} / \mathrm{ml}$ doxycycline

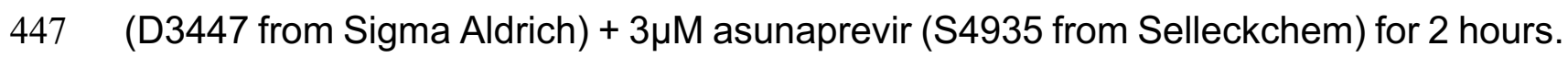


448 Then, $500 \mu \mathrm{M}$ auxin (I5148 from Sigma Aldrich) was added to the cell culture medium 449 for at least 4 hours. This approach was used in Fig1K; Fig. 3A-K; Extended data Fig. 450 2A; Extended data Fig. 6A-B.

451 Cytokinesis failure using drugs: cells were incubated with 10 $\mu \mathrm{M}$ genistein (G6649 452 from Sigma Aldrich) for at least two hours. This approach was used in Fig. 1E-F; 453 Extended data Fig. 1DF; Extended data Fig. 2D-E; Extended data Fig. 3A and F; 454 Extended data Fig. 3I-J; Extended data Fig. 6l. Alternatively, cell were incubated with $455 \quad 0.75 \mu \mathrm{M}$ Dihydrocytochalasin D (D1641 from Sigma-ALdrich) for 1 hour. This approach 456 was used in Fig. 2J; Extended data Fig. 2B; Extended data Fig. 4Q.

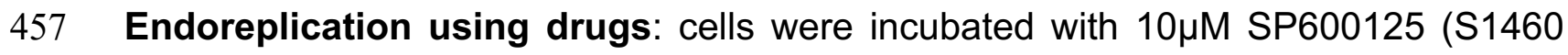
458 from Selleckchem) for at least two hours. This approach was used in Fig. 1G-H and 459 Extended data Fig. 1G-I.

460 Endoreplication using genetic tools: CCNA2 depletion in RPE CCNA2AID cells was 461 induced as described before ${ }^{49}$. Briefly, cells were treated with $2 \mu \mathrm{g} / \mathrm{ml}$ doxycycline 462 (D3447 from Sigma Aldrich) for 2 hours. Then, $500 \mu \mathrm{M}$ auxin (I5148 from Sigma 463 Aldrich) $+3 \mu \mathrm{M}$ asunaprevir (S4935 from Selleckchem) was added to the cell culture medium for at least 4 hours. This approach was used in Extended data Fig. 2C.

Cell cycle synchronization and DNA replication inhibition:

Cells were treated with $1 \mu \mathrm{M}$ palbociclib (Cdk4/6 inhibitor, S1579 from Selleckchem), with $0.5 \mu \mathrm{M}$ abemaciclib (Cdk4/6 inhibitor, S5716 from Selleckchem) or with $1 \mu \mathrm{M}$ K03861(Cdk2 inhibitor, S8100 from Selleckchem) for 16 hours to synchronize cells at G1/S transition and were collected (indicated by "G1 arrest" in the figures). Alternatively, cells were then washed five times using PBS 1X and released in S-phase for 10 hours before being collected (indicated by "Release in S-phase" in the figures). To inhibit DNA replication, cells were released in S-phase in the presence of low doses of Aphidicolin (APH, A0781 from Sigma-Aldrich), a DNA replication polymerase inhibitor, or of PHA767491 (PZ0178 from Sigma-Aldrich), a Cdc7 inhibitor (indicated by "Release in S-phase + APH" or "Release in S-phase + PHA", respectively, in the figures). Doses were chosen to significantly decrease EdU incorporation without affecting the levels of DNA damage. 


\begin{tabular}{|c|c|c|c|c|}
\hline Names: & Companies: & Targets: & References: & Concentrations: \\
\hline Auxin & Sigma & AID system & 15148 & $500 \mu \mathrm{M}$ \\
\hline Doxycycline & Sigma Aldrich & AID system & D3447 & $2 \mu \mathrm{g} / \mathrm{ml}$ \\
\hline Asunaprevir & Selleckchem & AID system & S4935 & $3 \mu \mathrm{M}$ \\
\hline Monastrol & Selleckchem & Eg5 & S8439 & $50 \mu \mathrm{M}$ \\
\hline MPI-0479605 & Selleckchem & MPS1 & S7488 & $1 \mu \mathrm{M}$ \\
\hline Genistein & Sigma Aldrich & MKLP1 & G6649 & $10 \mu \mathrm{M}$ \\
\hline SP600125 & Selleckchem & JNK & S1460 & $10 \mu \mathrm{M}$ \\
\hline Abemaciclib & Selleckchem & CDK4/6 & S5716 & $50 \mathrm{nM}$ or $0.5 \mu \mathrm{M}$ \\
\hline K03861 & Selleckchem & CDK2 & S8100 & $400 \mathrm{nM}$ or $1 \mu \mathrm{M}$ \\
\hline Palbociclib & Selleckchem & CDK4/6 & S1579 & $120 \mathrm{nM}$ or $1 \mu \mathrm{M}$ \\
\hline Aphidicolin & Sigma Aldrich & $\begin{array}{c}\text { DNA } \\
\text { polymerase }\end{array}$ & A0781 & $0.4 \mu \mathrm{M}$ or $1 \mu \mathrm{M}$ \\
\hline Hydroxyurea & Selleckchem & RNR & S1896 & $2 \mathrm{mM}$ \\
\hline PHA 767491 & Sigma Aldrich & Cdc7 & PZ0178 & $1 \mu \mathrm{M}$ \\
\hline RO3306 & Calbiochem & CDK1 & 217699 & $10 \mu \mathrm{M}$ \\
\hline $\begin{array}{c}\text { Dihydrocytochalasin } \\
\text { D }\end{array}$ & Sigma Aldrich & Actin & D1641 & $0.75 \mu \mathrm{M}$ \\
\hline $\begin{array}{c}\text { 5'-Chloro-2'- } \\
\text { deoxyuridine (CldU) }\end{array}$ & Sigma Aldrich & DNA & C6891 & $100 \mu \mathrm{M}$ \\
\hline $\begin{array}{c}\text { 5'-lodo-2'- } \\
\text { deoxyuridine (IdU) }\end{array}$ & Sigma Aldrich & DNA & 17125 & $100 \mu \mathrm{M}$ \\
\hline
\end{tabular}

\section{Fly husbandry and fly stocks:}

483 Flies were raised on cornmeal medium $(0.75 \%$ agar, 3.5\% organic wheat flour, $5.0 \%$ 484 yeast, $5.5 \%$ sugar, $2.5 \%$ nipagin, $1.0 \%$ penicillin-streptomycin and $0.4 \%$ propionic 485 acid). Fly stocks were maintained at $18^{\circ} \mathrm{C}$. Crosses were carried out in plastic vials and maintained at $25^{\circ} \mathrm{C}$. Stocks were maintained using balancer inverted chromosomes to prevent recombination. Stocks used in this study: sqh1 ${ }^{52}$, pavarotti RNAi (BL\#42573 from Bloomington Drosophila Stock Center, Indiana University, IN, USA) ${ }^{39}$, UAS-E2F1 (F001065 from FlyORF, Zurich, Switzerland) and UAS-Rb (BL\#

49050746 from Bloomington Drosophila Stock Center, Indiana University, IN, USA).

491 In all experiments, larvae were staged to obtain comparable stages of development.

492 Egg collection was performed at $25^{\circ} \mathrm{C}$ for 24 hours. After development at $25^{\circ} \mathrm{C}$, third 493 instar larvae were used for dissection. 
Immunofluorescence microscopy and antibodies:

Preparation and imaging of human cells

497 Cells were plated on cover slips in 12-well plates and treated with the indicated drugs. To label cells, they were fixed using $4 \%$ of paraformaldehyde (15710 from Electron Microscopy Sciences) + Triton X-100 (2000-C from Euromedex) 0,1\% in PBS (20 min at $4^{\circ} \mathrm{C}$ ). Then, cells were washed three times using PBS-T (PBS $1 \mathrm{X}+0,1 \%$ Triton $\mathrm{X}$ $100+0,02 \%$ Sodium Azide) and incubated with PBS-T + BSA (04-100-812-C from Euromedex) $1 \%$ for 30 min at RT. After three washes with PBS-T + BSA, primary and secondary antibodies were incubated in PBS-T + BSA $1 \%$ for $1 \mathrm{hr}$ and $30 \mathrm{~min}$ at RT, respectively. After two washes with PBS, cells were incubated with $3 \mu \mathrm{g} / \mathrm{ml}$ DAPI $(4,6$ diamidino-2-phenylindole; D8417 from Sigma Aldrich) for $15 \mathrm{~min}$ at RT. After two washes with PBS slides were mounted using 1.25\% n-propyl gallate (Sigma, P3130), 75\% glycerol (bidistilled, 99.5\%, VWR, 24388-295), 23.75\% H2O.

Images were acquired on an upright widefield microscope (DM6B, Leica Systems, Germany) equipped with a motorized XY and a 40X objective (HCX PL APO 40X/1,400,70 Oil from Leica). Acquisitions were performed using Metamorph software

512 (Molecular Devices, USA) and a sCMOS camera (Flash 4V2, Hamamatsu, Japan).

513 Stacks of conventional fluorescence images were collected automatically at a Z514 distance of $0.5 \mu \mathrm{m}$ (Metamorph software; Molecular Devices, RRID:SCR_002368). 515 Images are presented as maximum intensity projections generated with ImageJ 516 software (RRID:SCR_002285).

Whole mount tissue preparation and imaging of Drosophila larval brains

519 Brains or Salivary glands from third instar larvae were dissected in PBS and fixed for 52030 minutes in 4\% paraformaldehyde in PBS. They were washed three times in PBST $5210.3 \%$ (PBS, 0.3\% Triton X-100 (T9284, Sigma), 10 minutes for each wash) and 522 incubated for several hours in agitation at room temperature (RT) and $\mathrm{O} / \mathrm{N}$ at $4^{\circ} \mathrm{C}$ with 523 primary antibodies at the appropriate dilution in PBST $0.3 \%$. Tissues were washed 524 three times in PBST 0.3\% (10 minutes for each wash) and incubated $\mathrm{O} / \mathrm{N}$ at $4{ }^{\circ} \mathrm{C}$ with 525 secondary antibodies diluted in PBST 0.3\%. Brains and salivary glands were then 526 washed two times in PBST 0.3\% (30 minutes for each wash), rinsed in PBS and 527 incubated with $3 \mathrm{\mu g} / \mathrm{ml}$ DAPI (4',6-diamidino-2-phenylindole; D8417 from Sigma 
Aldrich) at RT for 30min. Brains and salivary glands were then washed in PBST 0.3\% at RT for 30 minutes and mounted in mounting media. A standard mounting medium was prepared with 1.25\% n-propyl gallate (Sigma, P3130), 75\% glycerol (bidistilled, 99.5\%, VWR, 24388-295), 23.75\% H2O.

533 Images were acquired on a spinning disk microscope (Gataca Systems, France). 534 Based on a CSU-W1 (Yokogawa, Japan), the spinning head was mounted on an 535 inverted Eclipse Ti2 microscope equipped with a motorized XY Stage (Nikon, Japan). 536 Images were acquired through a 40X NA 1.3 oil objective with a sCMOS camera 537 (Prime95B, Photometrics, USA). Optical sectioning was achieved using a piezo stage 538 (Nano-z series, Mad City Lab, USA). Gataca Systems' laser bench was equipped with 405, 491 and $561 \mathrm{~nm}$ laser diodes, delivering $150 \mathrm{~mW}$ each, coupled to the spinning disk head through a single mode fibre. Multi-dimensional acquisitions were performed using Metamorph 7.10.1 software (Molecular Devices, USA). Stacks of conventional

542 fluorescence images were collected automatically at a Z-distance of $1.5 \mu \mathrm{m}$ 543 (Metamorph software; Molecular Devices, RRID:SCR_002368). Images are presented 544 as maximum intensity projections generated with ImageJ software 545 (RRID:SCR_002285).

\section{Primary and secondary antibodies}

548 Primary and secondary antibodies were used at the following concentrations: Guinea 549 pig anti CEP192 antibody (1/500; Basto lab) ${ }^{53}$, rabbit anti beta catenin (1/250; C2206 550 from Sigma-Aldrich, RRID:AB_476831), mouse anti-gamma H2A.X phospho S139 (1/1000; ab22551 from Abcam, RRID:AB_447150), mouse anti-XRCC1 (1/500; ab1838 from Abcam, RRID:AB_302636), rabbit anti-Rad51 (1/500; ab133534 from Abcam, RRID:AB_2722613), mouse anti-KU80 (1/200; MA5-12933 from

554 ThermoFisher, RRID:AB_10983840), rabbit anti-FANCD2 (1/150; NB100-182SS from 555 Novusbio, RRID:AB_1108397), rabbit anti- $\gamma \mathrm{H} 2 A v(1 / 500 ; 600-401-914$ from Rockland; 556 RRID: AB_11183655), Alexa Fluor® 647 Phalloidin (1/250; A22287 from 557 ThermoFisher Scientific, RRID:AB_2620155), goat anti-Rabbit IgG (H+L) Highly 558 Cross-Adsorbed Secondary Antibody, Alexa Fluor 647 (1/250; A21245 from 559 ThermoFisher, RRID:AB_2535813), Goat anti-Guinea Pig IgG (H+L) Highly Cross560 Adsorbed Secondary Antibody, Alexa Fluor 488 (1/250; A11073 from ThermoFisher, 561 RRID:AB_253411), Goat anti-Mouse lgG (H+L) Cross-Adsorbed Secondary Antibody, 
562 Alexa Fluor 546 (1/250, A11003 from ThermoFisher, RRID:AB_2534071), Goat anti-

563 Rabbit IgG $(\mathrm{H}+\mathrm{L})$ Highly Cross-Adsorbed Secondary Antibody, Alexa Fluor 546 (1/250;

564 A-11035 from Thermo Fisher Scientific, RRID:AB_2534093).

566 Quantitative analysis of DNA damage:

567 Analysis of Drosophila NBs

568 Staged 3rd instar larval brains were dissected, stained and imaged using the procedures described above. We used the $\gamma \mathrm{H} 2 \mathrm{Av}$ primary antibody, which was preferentially detected using a secondary antibody conjugated Alexa Fluor 546. We used this secondary antibody because it was found to provide the best signal to noise

572 ratio.

573 Quantitative analysis of DNA damage was carried out as previously described ${ }^{39}$. In brief, DNA damage was assessed using a $\gamma \mathrm{H} 2 \mathrm{Av}$ primary antibody detected with an Alexa Fluor secondary antibody. Confocal volumes were obtained with optical sections at $1.5 \mu \mathrm{m}$ intervals. Image analysis was performed using Fiji and a custom plugin developed by QUANTACELL. After manual segmentation of the nuclei, a thresholding operation was used to determine the percentage of $\gamma \mathrm{H} 2 \mathrm{Av}$ positive pixels (coverage) and their average intensity in a single $z$ plane in the center of the nucleus. Coverage and intensity were multiplied to obtain the $\gamma \mathrm{H} 2 \mathrm{Av}$ index.

Analysis of human cell lines

583 For DNA damage quantification, the signals obtained in cultured cells were different

584 from the signals found in Drosophila NBs. To asses DNA damage in human cells, we used an ImageJ software-based plugin developed by QUANTACELL, where $\gamma \mathrm{H} 2 \mathrm{AX}$ signals were measured using z-projection stacks after thresholding. Both $\mathrm{FI}$ and the percentage of nuclear coverage was obtained for each nucleus. $\gamma \mathrm{H} 2 \mathrm{AX}$ index was obtained multiplying FI by the coverage.

590 All data plotting and statistical analyses were performed using the GraphPad Prism 591 software.

\section{Time lapse microscopy:}


594 Cells were plated on a dish (627870 from Dutscher) and treated with the indicated 595 drug. Images were acquired on a spinning disk microscope (Gataca Systems, France).

596 Based on a CSU-W1 (Yokogawa, Japan), the spinning head was mounted on an 597 inverted Eclipse Ti2 microscope equipped with a motorized XY Stage (Nikon, Japan). 598 Images were acquired through a 40X NA 1.3 oil objective with a sCMOS camera 599 (Prime95B, Photometrics, USA). Optical sectioning was achieved using a piezo stage 600 (Nano-z series, Mad City Lab, USA). Gataca Systems' laser bench was equipped with 601405,491 and $561 \mathrm{~nm}$ laser diodes, delivering $150 \mathrm{~mW}$ each, coupled to the spinning disk head through a single mode fiber. Multi-dimensional acquisitions were performed using Metamorph 7.10.1 software (Molecular Devices, USA). Stacks of conventional

604 fluorescence images were collected automatically at a Z-distance of $0.5 \mu \mathrm{m}$ 605 (Metamorph software; Molecular Devices, RRID:SCR_002368). Images are presented 606 as maximum intensity projections generated with ImageJ software 607 (RRID:SCR_002285), from stacks deconvolved with an extension of Metamorph 608 software.

609

610 EdU staining:

611 EdU incorporation into DNA was visualized with the Click-it EdU imaging kit (C10338 612 from Life Technologies), according to the manufacturer's instructions. EdU was used 613 at a concentration of $1 \mu \mathrm{M}$ (Extended data Fig. $4 \mathrm{~N}$ and $6 \mathrm{E}$ ) or $10 \mu \mathrm{M}$ (Extended data 614 Fig. 4A and I) for the indicated time. Cells were incubated with the Click-it reaction 615 cocktail for 15 minutes.

616

617 FACS sorting of diploid and tetraploid cells:

618 A mix of diploid and tetraploid cells (see "generation of tetraploid cells" section) were 619 incubated with $2 \mu \mathrm{g} / \mathrm{ml}$ Hoescht (94403 from Sigma Aldrich) for 1 hour at $37^{\circ} \mathrm{C}, 5 \%$ $620 \mathrm{CO}_{2}$. Then, a single cell suspension was generated. Cells were washed using PBS 1X, 621 the supernatant was removed and cells were resuspended in cold cell culture medium 622 at $1 \times 10^{7}$ cell per $\mathrm{ml}$ and kept at $4^{\circ} \mathrm{C}$ during all the experiment. FACS sorting was 623 performed using Sony SH800 FACS (BD FACSDiva Software Version 8.0.1). 624 Compensation was performed using the appropriate negative control samples. 625 Experimental samples were then recorded and sorted using gating tools to select the 626 populations of interest. RFP+ / GFP- negative cells (G1 cells) were first selected. Then, 627 in this population, DNA content was used to segregate diploid (2n) and tetraploid (4n) 
628 G1 cells. Once gates have been determined, diploid and tetraploid G1 cells were 629 sorted into external collection tubes. Post-sort analysis was performed to determine 630 the purity of the sorted populations (see Extended data Fig. 6A).

\section{E2F1 overexpression:}

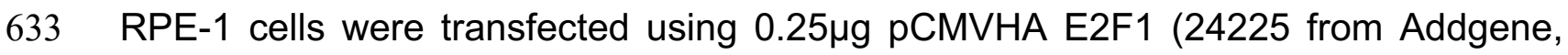
634 RRID:Addgene_24225) using JET PRIME kit (Polyplus Transfection, 114-07) 635 according to the manufacturer's protocol. Five hours later, cells were incubated with 636 DMSO (D8418 from Sigma Aldrich) or with 50 $\mu \mathrm{M}$ monastrol (S8439 from Selleckchem) $637+1 \mu \mathrm{M}$ MPI-0479605 (S7488 from Selleckchem) to generate tetraploid cells. After 2 638 hours, DMSO or $1 \mu \mathrm{M}$ palbociclib (S1579 from Sellechem) were added to the cell 639 culture medium for 16 hours. Cells were then fixed in G1 (T0) or washed five times 640 using PBS and released in S-phase and fixed after 10 hours (T10). The 641 immunofluorescence protocol is described in the corresponding section.

642 pCMVHA E2F1 was a gift from Kristian Helin (Addgene plasmid \# 24225 ; http://n2t.net/addgene:24225 ; RRID:Addgene_24225) ${ }^{54}$.

\section{Western Blot analysis and antibodies:}

646 Cells were lysed in $8 \mathrm{M}$ urea, $50 \mathrm{mM}$ Tris $\mathrm{HCl}, \mathrm{pH} 7.5$ and $150 \mathrm{mM} \beta$-mercaptoethanol 647 (161-0710 from Bio-Rad), sonicated and heated at $95^{\circ} \mathrm{C}$ for 10 minutes. Samples 648 (equivalent of $2 \times 10^{5}$ cells) were subjected to electrophoresis in NuPAGE Novex 4649 12\% Bis-Tris pre-cast gels (NP0321 from Life Technologies). Protein fractions from the 650 gel were electrophoretically transferred to PVDF membranes (PVDF transfer 651 membrane; RPN303F from GE). After $1 \mathrm{hr}$ saturation in PBS containing $5 \%$ dry non652 fat milk and $0.5 \%$ Tween 20 , the membranes were incubated for $1 \mathrm{hr}$ with a primary 653 antibody (see below) diluted in PBS containing 5\% dry non-fat milk and 0.5\% Tween 654 20. After three 10-min washes with PBS containing 0.5\% Tween 20, the membranes 655 were incubated for 45 min with a 1/2 500 dilution of peroxidase-conjugated antibody 656 (see below). Membranes were then washed three times with PBS containing 0.5\% 657 Tween 20, and the reaction was developed according to the manufacturer's 658 specifications using ECL reagent (SuperSignal West Pico Chemiluminescent 659 Substrate; 34080 from Thermo Scientific). Protein levels were normalized using H2B 660 signal and quantifications were done using Image Lab software version 6.0.1, Bio-Rad 661 Laboratories. 
663 Primary and secondary antibodies were used at the following concentrations:

664 Mouse anti Tubulin (1/5000; T9026 from Sigma, RRID:AB_477593), mouse anti 665 CDC45 (1/500; sc-55569 from Santa Cruz Biotechnology, RRID:AB_831146), rabbit 666 anti PCNA (1/500; sc56 from Santa Cruz, RRID:AB_628110), rabbit anti Actin (1/2000; 667 A5060 from Sigma-Aldrich, RRID:AB_476738), mouse anti-H2B (1/1000; sc-515808 668 from Santa Cruz Biotechnology), mouse anti ORC1 (1/500; sc-398734 from Santa 669 Cruz Biotechnology), mouse anti MCM2 (1/500; 610701 from BD Biosciences, 670 RRID:AB_398024), Goat anti-Rabbit lgG (H+L) Cross-Adsorbed Secondary Antibody, 671 HRP (1/2500; G21234 from ThermoFisher, RRID:AB_2536530), Peroxidase AffiniPure 672 Goat Anti-Mouse IgG $(\mathrm{H}+\mathrm{L})(1 / 2500$; 115-035-003 from Jackson ImmunoResearch, 673 RRID:AB_10015289).

\section{D reconstruction and analysis on Imaris:}

676 3D movies (see time lapse microscopy section) were imported into Imaris software 677 v.9.6.0 (Bitplane, RRID:SCR_007370). For chosen cells, the module "Spot tracking" of 678 Imaris was used to detect the foci, as spots of diameter $0.5 \mu \mathrm{m}$ in the XY-direction and $6791 \mu \mathrm{m}$ in Z-direction (modelling PSF elongation). Because the volume of the foci 680 changes in time, the option "Enable growing regions" was used. In each movie, the 681 threshold was chosen on the brightest frame (to detect a maximum of the correct spots) 682 and then applied to the whole movie. For each cell, at each time point, the number of 683 spots and volumes were recorded. For each condition, at least 10 cells were studied 684 and the statistics from Imaris were averaged at each time point using a MATLAB script. 685

\section{Molecular combing and antibodies:}

688 dihydrocytochalasin D (DCD, inhibitor of actin polymerization, D1641 from Sigma689 Aldrich) for $18 \mathrm{~h}$ overnight. Afterwards, the cells were washed three times with PBS 690 and cultured in DMEM supplemented with 10\% FBS and 1\% Pen/Strep for additional $69110 \mathrm{~h}$. Cells were pulse-labelled with $0.1 \mathrm{mM} \mathrm{CldU}$ and $0.1 \mathrm{mM}$ IdU for 30min and 100 692000 cells per condition were collected for further analysis. The DNA was extracted from 693 cells and prepped following the manufacturer's instructions using the FiberPrep® DNA 694 Extraction Kit (Genomic Vision, Bagneux, France). Subsequently, the prepped DNA 695 was stretched onto coated glass coverslips (CombiCoverslips ${ }^{\mathrm{TM}}$, Genomic Vision, 
696 Bagneux, France) by using the FiberComb Molecular Combing System (Genomic

697 Vision, Bagneux, France). The Labelling was performed with antibodies against

698 ssDNA, IdU and CldU using the Replication Combing Assay (RCA) (Genomic Vision,

699 Bagneux, France). The imaging of the prepared cover slips was carried out by

700 Genomic Vision (Bagneux, France) and analysed using the FiberStudio ${ }^{2}$ 2.0.1

701 Analysis Software by Genomic Vision.

702

703 Antibodies were used at the following concentrations:

704 Rabbit anti ssDNA (1/5; 18731 from IBL International, RRID:AB_494649), Rat anti

705 CldU (1/10; Ab6326 from Abcam, RRID:AB_2313786), Mouse anti IdU (1/10; 555627

706 from BD Biosciences, RRID:AB_10015222), mouse Alexa Fluor 647 Donkey (1/25;

707 JIM-715-605-151 from Biozol), Rat Alexa Fluor 594 Donkey (1/25; JIM-712-585-153

708 from Biozol), Rabbit Brilliant Violet 480 Donkey (1/25; 711-685-152 from Jakcson

709 Immuno Research, RRID:AB_2651109).

710

711 Quantitative phase imaging and measurements

712 Cells were plated on glass-bottom dishes coated with $50 \mu \mathrm{g} / \mathrm{ml}$ Fibronectin for 1 hour 713 and rinsed, and trypsinised cells were plated at a concentration of $1.5^{\star} 10^{6} \mathrm{cells} / \mathrm{ml}$. The 714 cells used for the experiments were seeded in T-25 dishes at a concentration of $0.7^{*} 10^{6}$ 715 cells $/ \mathrm{ml} 2$ days before the actual experiment. On the day of the experiment, the cells 716 were detached with EDTA (versene), and plated at a concentration of $1.5^{\star} 10^{6} \mathrm{cells} / \mathrm{ml}$.

717 For inducing tetraploidy, cells were treated with $2 \mu \mathrm{g} / \mathrm{ml}$ doxycycline (D3447 from Sigma

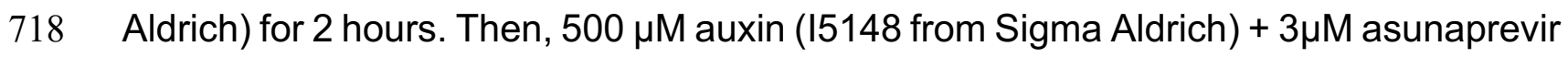
719 (S4935 from Selleckchem) was added to the cell culture medium for at least 4 hours.

720 The cells were then imaged for 35 hours every 20 minutes to track them throughout 721 their cell cycle.

722 The cell cycle state of the cells was indicated by the FUCCI system; G1 cells express 723 Cdt1-RFP while S/G2 cells express hGeminin-GFP and mitosis is indicated by the 724 NEBD with geminin being present through the cells ${ }^{55}$. To quantify the fluorescence of 725 geminin in the nucleus, first a background subtraction was performed on the images. 726 An ROI was used to define an area containing the background fluorescence in the 727 image. An average value of the ROI was then subtracted from all the frames. 728 Subsequently, a ROI was drawn as close as possible to the cell, and then the mean 
gray value was measured across all the frames. This helped identify the frames of birth and $\mathrm{G} 1 / \mathrm{S}$ transition during cell cycle.

A detailed protocol for the mass measurement with phasics camera is available in ${ }^{56,57}$. Images were acquired by Phasics camera every 20 min for 35 hours for the duration of the experiment. To obtain the reference image, 32 empty fields were acquired on the dish and a median image was calculated. This reference image was subtracted from the interferograms (images acquired by phasics) by custom written MATLAB scripts to measure the optical path difference. They were then processed to calculate the phase, intensity and phase cleaned images (the background set to 1000 and the field cropped to remove edges). Background normalization was performed using a gridfit method and a watershed algorithm was used to separate cells which came in contact with each other. Mass was calculated by integrating the intensity of the whole cell.

\section{Sequencing and AneuFinder analysis:}

A mixed population of diploid and tetraploid RPE-1 CCNB1AID FUCCI cells were synchronized in G1 using 1 $\mu \mathrm{M}$ palbociclib (S1579 from Selleckchem) for 16 hours or

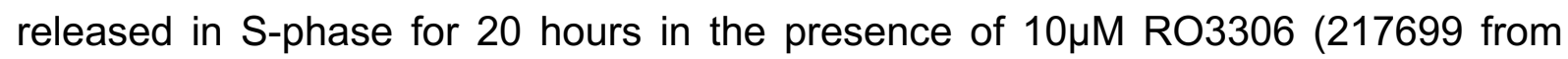
Calbiochem) in order to block cells in the subsequent G2/M. G1 and G2/M diploid and tetraploid cells were then isolated using cell sorting (see "FACS sorting of diploid and tetraploid cells" section) and collected in a 96-well plate. Single-cell sequencing was performed as described in detail in ${ }^{58}$. Briefly, cells were lysed to prepare a suspension of nuclei and sorted as single nuclei in 96 or 384 well plates. Next, single cell sequencing libraries were prepared using a semi-automated liquid handler platform (Bravo, Agilent technologies). For library preparation, chromatin was fragmented by

754 micrococcal nuclease, end-repaired, and A-tailed, followed by Illumina adapter ligation. Libraries were then cleaned up and PCR-amplified for 17 cycles that included the addition of a library-specific barcode to uniquely label individual cell libraries. Up to 384 libraries were pooled and sequenced on a Nextseq 500 machine (Illumina; up to 77 cycles; single end). The generated data were subsequently demultiplexed using sample-specific barcodes and changed into fastq files using bcl2fastq (Illumina; version 1.8.4). Reads were afterwards aligned to the human reference genome (GRCh38/hg38) using Bowtie2 (version 2.2.4) ${ }^{59}$. Duplicate reads were marked with 
number calling algarithm called AneuFinder (https://github.com/ataudt/aneufinder) ${ }^{61}$. Following GC correction and blacklisting of artefact-prone regions (extreme low or high coverage in control samples), libraries were analyzed using the dnacopy and edivisive copy number calling algorithms with variable width bins (average binsize $=1 \mathrm{Mb}$; step size $=500 \mathrm{~kb}$ ). The $\mathrm{G} 1$ samples were used as reference for the analysis of the G2/M samples (G1 diploid for G2/M diploid and $\mathrm{G} 1$ polyploid for $\mathrm{G} 2 / \mathrm{M}$ polyploid). The $\mathrm{G} 1$ samples were analyzed with an euploid reference ${ }^{62}$. Results were afterwards curated by requiring a minimum concordance of $90 \%$ (4N and $8 \mathrm{~N}$ samples) or $95 \%(2 \mathrm{~N}$ samples) between the results of the two algorithms. Libraries with on average less than 10 reads per bin ( $\sim 30,000$ reads for a diploid genome) were discarded. The aneuploidy scores corresponds to the absolute difference from euploid genome and is the average from all bins and all libraries of one sample. Heterogeneity scores is calculated as the proportion of pairwise comparisons, between libraries, that shows different copy numbers. This is first calculated for each bin. To get to the final score a weighted average is applied.

QUANTIFICATION AND STATISTICAL ANALYSIS:

\section{Quantifications:}

782 Image analysis and quantifications were performed using Image $\mathrm{J}$ software V2.1.0/1.53c, https://imagej.net/software/fiji/downloads. To quantify the colocalizations between two signals (Extended data Fig. 3M and 4D) we used JACOP plugin with Image J software. 3D movies (Fig. 2F and Extended data Fig. 3B) were corrected using $3 D$ correct drift plugin with Image $\mathrm{J}$ software to keep the cell of interest at the centre of the region of interest. The nuclear area and DAPI intensity were measured using the wand tool with Image $\mathrm{J}$ software. For the figures, images were processed on Image $\mathrm{J}$ software, and mounted using Affinity Designer, https://affinity.serif.com/fr/designer/.

Statistical analysis:

At least three $(n)$ independent experiments were carried out to generate each dataset, and the statistical significance of differences was calculated with Student's t-test.

794 These tests were performed using GraphPad Prism (RRID:SCR_002798) version 7.00 for Mac, GraphPad Software, La Jolla California USA, www.graphpad.com. 
bioRxiv preprint doi: https://doi.org/10.1101/2021.07.16.452672; this version posted July 16, 2021. The copyright holder for this preprint (which was not certified by peer review) is the author/funder. All rights reserved. No reuse allowed without permission. 


\section{REFERENCES:}

799

800 1. Zack, T. I. et al. Pan-cancer patterns of somatic copy number alteration. Nat.

801

802

803

804

805

806

807

808

809

810

811

812

813

814

815

816

817

818

819

820

821

822

823

824

825

826

827

828

829

830

831

Genet. 45, 1134-1140 (2013).

2. Bielski, C. M. et al. Genome doubling shapes the evolution and prognosis of advanced cancers. Nat. Genet. 50, 1189-1195 (2018).

3. López, S. et al. Interplay between whole-genome doubling and the accumulation of deleterious alterations in cancer evolution. Nat. Genet. 52, 283-293 (2020).

4. Storchova, Z. \& Kuffer, C. The consequences of tetraploidy and aneuploidy. Journal of Cell Science vol. 121 3859-3866 (2008).

5. Storchova, Z. \& Pellman, D. From polyploidy to aneuploidy, genome instability and cancer. Nature Reviews Molecular Cell Biology vol. 5 45-54 (2004).

6. Dewhurst, S. M. et al. Tolerance of whole- genome doubling propagates chromosomal instability and accelerates cancer genome evolution. Cancer Discov. 4, 175-185 (2014).

7. Fox, D. T., Gall, J. G. \& Spradling, A. C. Error-prone polyploid mitosis during normal Drosophila development. Genes Dev. 24, 2294-2302 (2010).

8. Goupil, A. et al. Chromosomes function as a barrier to mitotic spindle bipolarity in polyploid cells. J. Cell Biol. 219, (2020).

9. Crockford, A. et al. Cyclin D mediates tolerance of genome-doubling in cancers with functional p53. Ann. Oncol. 28, 149-156 (2017).

10. Potapova, T. A., Seidel, C. W., Box, A. C., Rancati, G. \& Li, R. Transcriptome analysis of tetraploid cells identifes cyclin D2 as a facilitator of adaptation to genome doubling in the presence of p53. Mol. Biol. Cell 27, 3065-3084 (2016).

11. Andreassen, P. R., Lohez, O. D., Lacroix, F. B. \& Margolis, R. L. Tetraploid state induces p53-dependent arrest of nontransformed mammalian cells in G1. Mol. Biol. Cell 12, 1315-1328 (2001).

12. Storchová, Z. et al. Genome-wide genetic analysis of polyploidy in yeast. Nature 443, 541-547 (2006).

13. Carter, S. L. et al. Absolute quantification of somatic DNA alterations in human cancer. Nat. Biotechnol. 30, 413-421 (2012).

14. Ganem, N. J., Storchova, Z. \& Pellman, D. Tetraploidy, aneuploidy and cancer. Current Opinion in Genetics and Development vol. 17 157-162 (2007). 
15. Gemble, S. \& Basto, R. CHRONOCRISIS: When Cell Cycle Asynchrony Generates DNA Damage in Polyploid Cells. BioEssays 42, (2020).

16. Kuznetsova, A. Y. et al. Chromosomal instability, tolerance of mitotic errors and multidrug resistance are promoted by tetraploidization in human cells. Cell Cycle 14, 2810-2820 (2015).

17. Jemaà, M. et al. Whole-genome duplication increases tumor cell sensitivity to MPS1 inhibition. Oncotarget 7, 885-901 (2016).

18. Quinton, R. J. et al. Whole-genome doubling confers unique genetic vulnerabilities on tumour cells. Nature 590, 492-497 (2021).

19. Fujiwara, T. et al. Cytokinesis failure generating tetraploids promotes tumorigenesis in p53-null cells. Nature 437, 1043-1047 (2005).

20. Orr-Weaver, T. L. When bigger is better: The role of polyploidy in organogenesis. Trends in Genetics vol. 31 307-315 (2015).

21. Zeman, M. K. \& Cimprich, K. A. Causes and Consequences of Replication Stress. Nat. Cell Biol. 16, 2 (2014).

22. Koundrioukoff, S. et al. Stepwise activation of the ATR signaling pathway upon increasing replication stress impacts fragile site integrity. PLoS Genet. 9, e1003643 (2013).

23. Panier, S. \& Boulton, S. J. Double-strand break repair: 53BP1 comes into focus. Nat. Rev. Mol. Cell Biol. 15, 7-18 (2014).

24. Zhao, B., Rothenberg, E., Ramsden, D. A. \& Lieber, M. R. The molecular basis and disease relevance of non-homologous DNA end joining. Nat. Rev. Mol. Cell Biol. 21, 765-781 (2020).

25. A, B., T, L. \& A, C. Quantitative live imaging of endogenous DNA replication in mammalian cells. PLoS One 7, (2012).

26. Michalet, X. et al. Dynamic molecular combing: Stretching the whole human genome for high- resolution studies. Science (80-. ). 277, 1518-1523 (1997).

27. Zlotek-Zlotkiewicz, E., Monnier, S., Cappello, G., Berre, M. Le \& Piel, M. Optical volume and mass measurements show that mammalian cells swell during mitosis. J. Cell Biol. 211, 765 (2015).

28. Tatsumi, Y., Ohta, S., Kimura, H., Tsurimoto, T. \& Obuse, C. The ORC1 cycle in human cells: I. Cell cycle-regulated oscillation of human ORC1. J. Biol. Chem. 278, 41528-41534 (2003).

29. Remus, D. et al. Concerted Loading of Mcm2-7 Double Hexamers around DNA 
866

867

868

869

870

871

872

873

874

875

876

877

878

879

880

881

882

883

884

885

886

887

888

889

890

891

892

893

894

895

896

897

898

899

during DNA Replication Origin Licensing. Cell 139, 719-730 (2009).

30. Moyer, S. E., Lewis, P. W. \& Botchan, M. R. Isolation of the Cdc45/Mcm27/GINS (CMG) complex, a candidate for the eukaryotic DNA replication fork helicase. Proc. Natl. Acad. Sci. U. S. A. 103, 10236-10241 (2006).

31. Bertoli, C., Skotheim, J. M. \& De Bruin, R. A. M. Control of cell cycle transcription during $\mathrm{G} 1$ and S phases. Nature Reviews Molecular Cell Biology vol. 14 518-528 (2013).

32. Pardee, A. B. G1 events and regulation of cell proliferation. Science (80-. ). 246, 603-608 (1989).

33. Cadart, C. et al. Size control in mammalian cells involves modulation of both growth rate and cell cycle duration. Nat. Commun. 9, (2018).

34. DW, F. et al. Specific inhibition of cyclin-dependent kinase 4/6 by PD 0332991 and associated antitumor activity in human tumor xenografts. Mol. Cancer Ther. 3, 1427-1437 (2004).

35. Tan, C. et al. Cell size homeostasis is maintained by CDK4-dependent activation of p38 MAPK. Dev. Cell 56, 1756-1769.e7 (2021).

36. Charvin, G., Oikonomou, C., Siggia, E. D. \& Cross, F. R. Origin of irreversibility of cell cycle start in budding yeast. PLoS Biol. 8, (2010).

37. Cross, F. R., Buchler, N. E. \& Skotheim, J. M. Evolution of networks and sequences in eukaryotic cell cycle control. Philosophical Transactions of the Royal Society B: Biological Sciences vol. 366 3532-3544 (2011).

38. Zatulovskiy, E., Zhang, S., Berenson, D. F., Topacio, B. R. \& Skotheim, J. M. Cell growth dilutes the cell cycle inhibitor $\mathrm{Rb}$ to trigger cell division. Science (80-. ). 369, 466-471 (2020).

39. Nano, M. et al. Cell-Cycle Asynchrony Generates DNA Damage at Mitotic Entry in Polyploid Cells. Curr. Biol. 29, (2019).

40. Frawley, L. E. \& Orr-Weaver, T. L. Polyploidy. Current Biology vol. 25 R353R358 (2015).

41. Tseng, A.-S. K. \& Hariharan, I. K. An Overexpression Screen in Drosophila for Genes That Restrict Growth or Cell-Cycle Progression in the Developing Eye. (2002).

42. Duronio, R. J., Brook, A., Dyson, N. \& O'Farrell, P. H. E2F-induced S phase requires cyclin E. Genes Dev. 10, 2505-2513 (1996).

43. Maya-Mendoza, A. et al. High speed of fork progression induces DNA 
900

901

902

903

904

905

906

907

908

909

910

911

912

913

914

915

916

917

918

919

920

921

922

923

924

925

926

927

928

929

930

931

932

933

replication stress and genomic instability. Nature 559, 279-284 (2018).

44. Sedlackova, H. et al. Equilibrium between nascent and parental MCM proteins protects replicating genomes. Nature 587, 297-302 (2020).

45. M, T. et al. Functional reprogramming of polyploidization in megakaryocytes. Dev. Cell 32, 155-167 (2015).

46. Klusza, S. \& Deng, W.-M. At the crossroads of differentiation and proliferation: Precise control of cell-cycle changes by multiple signaling pathways in Drosophila follicle cells. Bioessays 33, 124 (2011).

47. Viganó, C. et al. Quantitative proteomic and phosphoproteomic comparison of human colon cancer DLD-1 cells differing in ploidy and chromosome stability. Mol. Biol. Cell 29, 1031-1047 (2018).

48. Wangsa, D. et al. Near-tetraploid cancer cells show chromosome instability triggered by replication stress and exhibit enhanced invasiveness. FASEB J. 32, 3502-3517 (2018).

49. Hégarat, N. et al. Cyclin A triggers Mitosis either via the Greatwall kinase pathway or Cyclin B. EMBO J. 39, (2020).

50. SB, K. et al. A quantitative FastFUCCI assay defines cell cycle dynamics at a single-cell level. J. Cell Sci. 130, 512-520 (2017).

51. KS, Y., RH, K., M, L., R, G. \& R, W. Single cell resolution in vivo imaging of DNA damage following PARP inhibition. Sci. Rep. 5, (2015).

52. Karess, R. E. et al. The regulatory light chain of nonmuscle myosin is encoded by spaghetti-squash, a gene required for cytokinesis in Drosophila. Cell 65, 1177-1189 (1991).

53. Gemble, S. et al. Centromere Dysfunction Compromises Mitotic Spindle Pole Integrity. Curr. Biol. 29, (2019).

54. J, L., BO, P., K, H., J, B. \& K, H. Deregulated expression of E2F family members induces S-phase entry and overcomes p16INK4A-mediated growth suppression. Mol. Cell. Biol. 16, 1047-1057 (1996).

55. Sakaue-Sawano, A. et al. Visualizing Spatiotemporal Dynamics of Multicellular Cell-Cycle Progression. Cell 132, 487-498 (2008).

56. Aknoun, S. et al. Living cell dry mass measurement using quantitative phase imaging with quadriwave lateral shearing interferometry: an accuracy and sensitivity discussion. J. Biomed. Opt. 20, 126009 (2015).

57. Bon, P., Maucort, G., Wattellier, B. \& Monneret, S. Quadriwave lateral shearing 
interferometry for quantitative phase microscopy of living cells. Opt. Express 17, 13080 (2009).

936

58. van den Bos, $\mathrm{H}$. et al. Quantification of aneuploidy in mammalian systems. in Methods in Molecular Biology vol. 1896 159-190 (Humana Press Inc., 2019).

59. Langmead, B. \& Salzberg, S. L. Fast gapped-read alignment with Bowtie 2. Nat. Methods 9, 357-359 (2012).

60. Jun, G., Wing, M. K., Abecasis, G. R. \& Kang, H. M. An efficient and scalable analysis framework for variant extraction and refinement from population-scale DNA sequence data. Genome Res. 25, 918-925 (2015).

943 61. Bakker, B. et al. Single-cell sequencing reveals karyotype heterogeneity in murine and human malignancies. Genome Biol. 17, (2016).

62. van den Bos, H. et al. Single-cell whole genome sequencing reveals no evidence for common aneuploidy in normal and Alzheimer's disease neurons. Genome Biol. 17, 1-9 (2016). 
Figure fioRxiv preprint doi: https://doi.org/10.1101/2021.07.16.452672; this version posted July 16, 2021. The copyright holder for this preprint (which was not certified by peer review) is the author/funder. All rights reserved. No reuse allowed without permission.

A

응

Mitotic

slippage (MS)

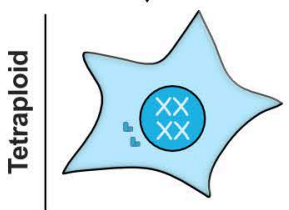

Whole genome duplication (WGD
B

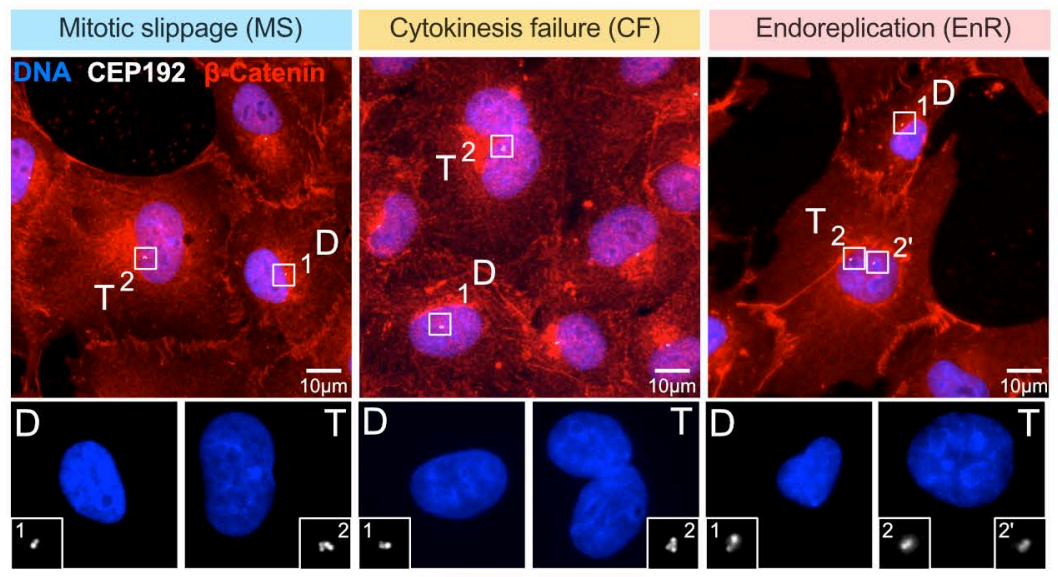

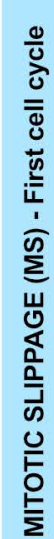

C

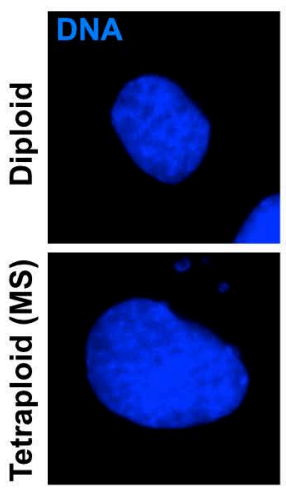

E
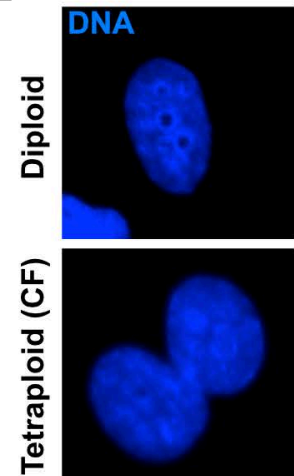

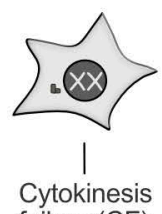

Cytokinesis
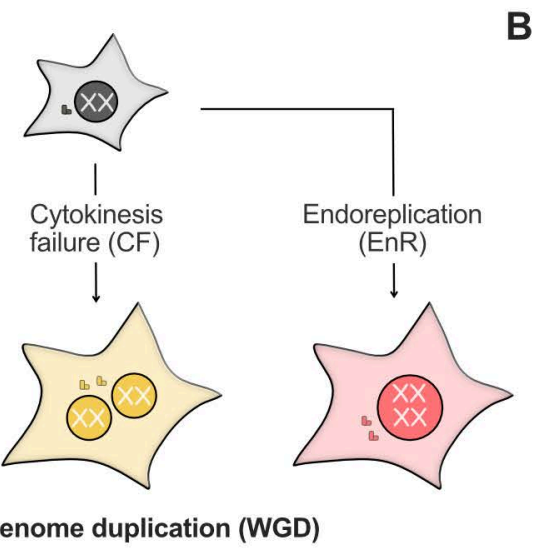
)

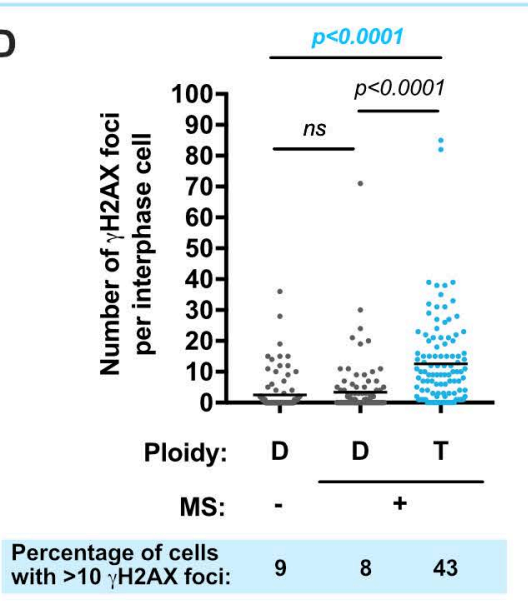

$\mathbf{F}$

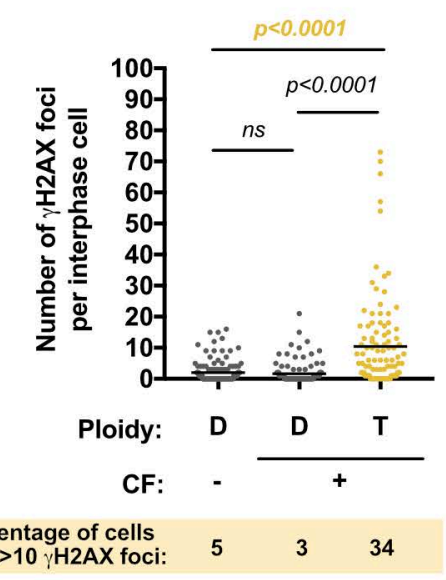

G

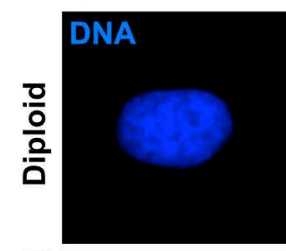

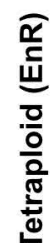
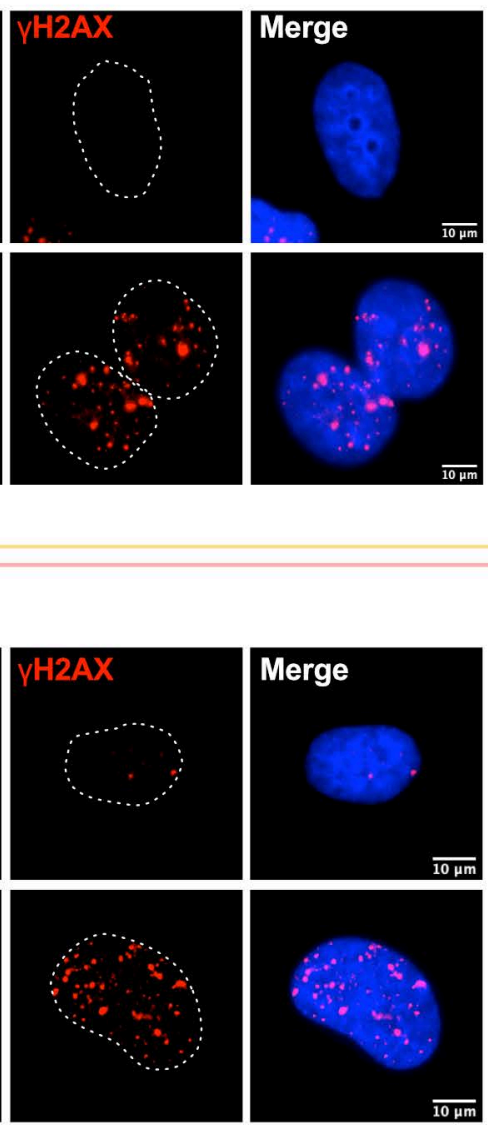

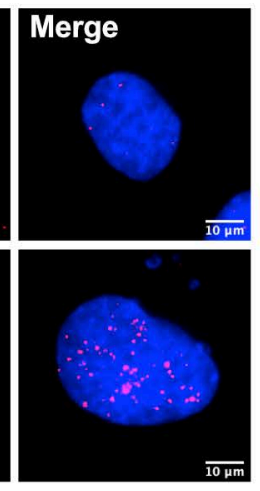

H

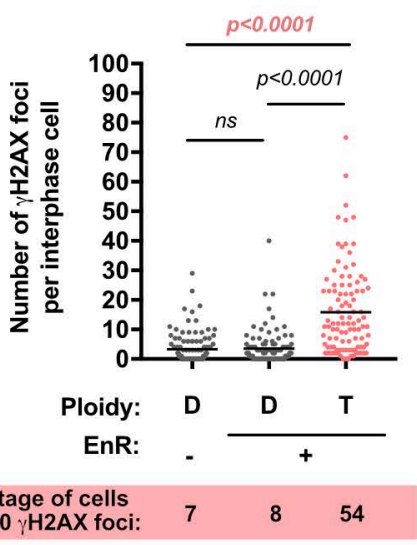

$\begin{array}{lllll}\begin{array}{l}\text { Percentage of cells } \\ \text { with }>10 \gamma \mathrm{H} 2 \mathrm{AX} \text { foci: }\end{array} & 7 & 8 & 54\end{array}$ 
Figure 1: High levels of DNA damage are generated in the first interphase following unscheduled WGD.

(A) Schematic representation of the generation of tetraploid cells. (B) Representative immunofluorescence images of diploid and tetraploid RPE-1 cells generated by inhibiting Eg5 and MPS1 (MS), or MKLP1 (CF), or JNK (EnR). DNA was visualized using DAPI (in blue), centrosomes were stained using anti-CEP192 antibodies (in white) and membranes were stained using anti- $\beta$-Catenin antibodies (in red). The white squares correspond to higher magnifications presented in the lower panel and showing the centrosomes (in yellow). (C, E and G) Representative immunofluorescence images showing DNA damage in diploid and tetraploid RPE-1 cells were generated by inhibiting Eg5 and MPS1 (MS), or MPS1 (CF), or JNK (EnR). DNA was visualized using DAPI (in blue), DNA damage was visualized using anti- $\gamma \mathrm{H} 2 \mathrm{AX}$ antibodies (in red). $>100$ interphase cells were analyzed from at least three independent experiments. (D, $\mathbf{F}$ and H) Graph showing the number of $\gamma \mathrm{H} 2 \mathrm{AX}$ foci per interphase cells in diploid and tetraploid RPE-1 cells. The percentage of interphase cells with more than $10 \gamma \mathrm{H} 2 \mathrm{AX}$ foci in diploid and tetraploid RPE-1 cells were indicated under the graph. $>100$ interphase cells were analyzed from at least three independent experiments. The dotted lines indicate the nuclear area. D: Diploid. T: Tetraploid. MS: mitotic slippage. CF: cytokinesis failure. EnR: endoreplication. 
Figure 2: DNA damage and genetic instability in tetraploid cells is generated during S-phase in a DNA replication-dependent manner.

(A) Upper panel - Representative immunofluorescence images showing DNA damage in RPE-1 tetraploid cells over time. DNA was visualized using DAPI (in blue), DNA damage is visualized using anti- $\gamma \mathrm{H} 2 \mathrm{AX}$ antibodies (in red). Lower panel - Percentage of RPE-1 FUCCI tetraploid cells in G1 (red) or in S-G2 (green) over time. (B) Graph representing the number of $\gamma \mathrm{H} 2 \mathrm{AX}$ foci per interphase cells in diploid (gray) and tetraploid (blue) RPE-1 cells over time. The percentage of interphase cells with more than $10 \gamma \mathrm{H} 2 \mathrm{AX}$ foci in diploid and tetraploid RPE-1 cells is indicated under the graph. $>100$ interphase cells were analyzed from at least three independent experiments. (C) Percentage of RPE-1 FUCCl tetraploid cells in G1 (red) or in S-G2 (green) and representative immunofluorescence images showing DNA damage in tetraploid cells synchronized in $\mathrm{G} 1$ using $1 \mu \mathrm{M}$ palbociclib or released in S-phase with or without 400nM aphidicolin (APH). DNA was visualized using DAPI (in blue), DNA damage was visualized using anti- $\gamma \mathrm{H} 2 \mathrm{AX}$ antibodies (in red). (D) Graph showing the number of $\gamma \mathrm{H} 2 \mathrm{AX}$ foci per interphase cells in diploid (gray) and tetraploid (blue) RPE-1 cells synchronized in $\mathrm{G} 1$ using $1 \mu \mathrm{M}$ palbociclib or released in S-phase with or without 400nM aphidicolin (APH). The percentage of interphase cells with more than $10 \mathrm{\gamma H} 2 \mathrm{AX}$ foci in diploid and tetraploid RPE-1 cells are indicated under the graph. $>100$ interphase cells were analyzed from at least three independent experiments. (E) Schematic workflow showing the method used in this study to process and analyze DNA replication by live imaging. (F) Stills of time lapse of diploid and tetraploid RPE-1 PCNA ${ }^{\text {chromo }}$ cells. Active replication sites are visualized using PCNA chromobodies (in cyan) and reconstructed using Imaris in 3D (in red). (G) Graph showing the total number of active replication sites during S-phase in diploid (gray) and tetraploid (blue) RPE-1 cells. >20 S-phase cells were analyzed from three independent experiments. (H) Graph showing the volume of active replication sites in $\mu \mathrm{m}^{3}$ in diploid (gray) and tetraploid (blue) RPE-1 PCNA ${ }^{\text {chromo }}$ cells. At least 1000 active replication sites were analyzed. (I) Graph showing the mean number of active replication sites over time in diploid (gray line) and tetraploid (blue line) RPE-1 cells. >20 S-phase cells were analyzed from three independent experiments. For other representative examples, see Figure S5A. (J) Left panel - Graph representing the replication fork speed in diploid (gray) and tetraploid (yellow) HCT116 cells. Right panel - Graph showing the percentage of unstable replication forks in diploid (gray) and tetraploid (yellow) HCT116 cells. More than 120 replication forks were analyzed. Lower panel Representative immunofluorescence of DNA fibers obtained from diploid and tetraploid HCT116 cells. ssDNA was visualized using anti-ssDNA antibodies (in blue), CldU and IdU was visualized using anti-IdU and anti-CldU antibodies (in red and green), respectively. (K) Genome-wide copy number plots G2/M tetraploid RPE-1 cells were generated using a modified version of the Aneufinder algorithm and normalized using G1 tetraploid cells (see methods). Each row represents a cell and the copy number state (in 5-Mb bins) is indicated in colors (with aberrations contrasting from from dark green in G2/M (8n). The dotted lines indicate nuclear area. D: Diploid. T: Tetraploid. MS: mitotic slippage. CF: cytokinesis failure. EnR: endoreplication. 
A

$\square \mathrm{G} 1 \square \mathrm{S}-\mathrm{G} 2$

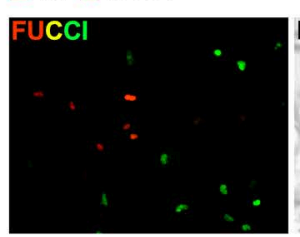

G1

rOP

G1/S transition

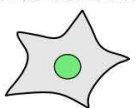

$\begin{gathered}\text { Amount of protein } \\ \text { produced during } \mathbf{G 1}\end{gathered}=\frac{\text { Cell mass at G1/S transition }}{\text { Cell mass at G1 entry }}$

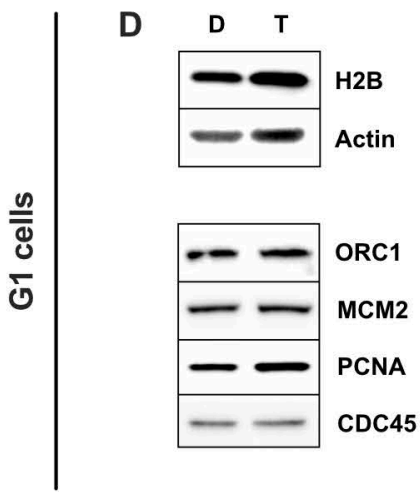

B

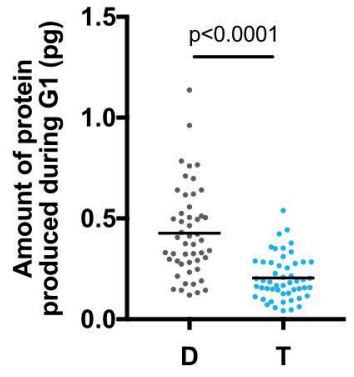

MS:

E

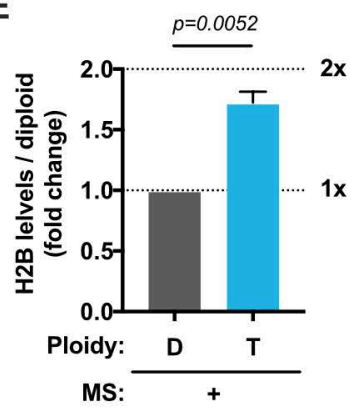

C

G1 population expressing FUCCI

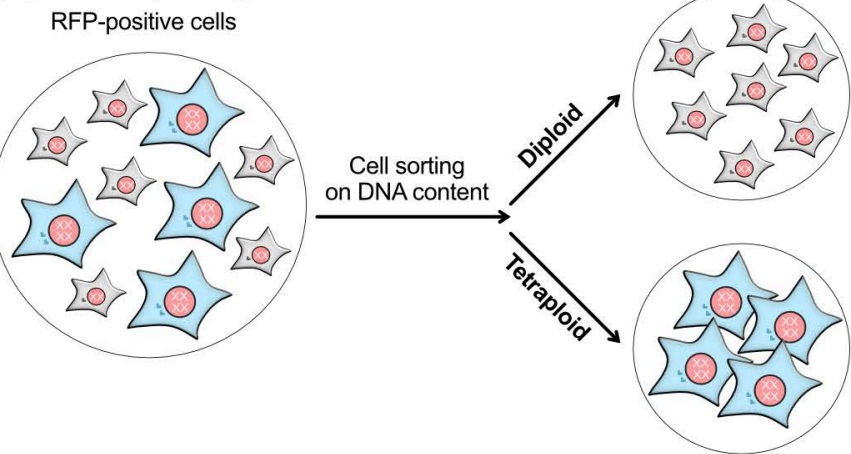

F

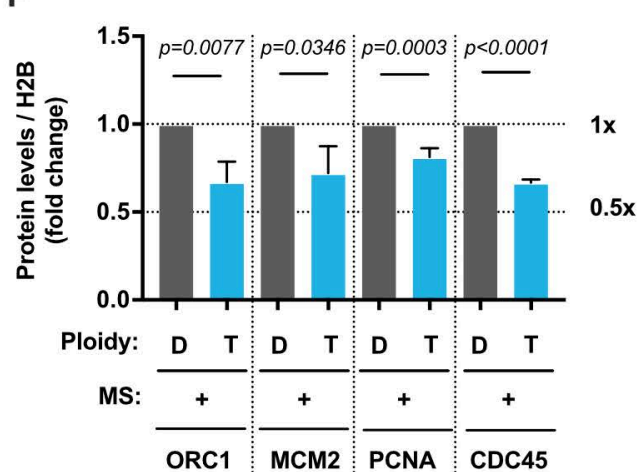

G

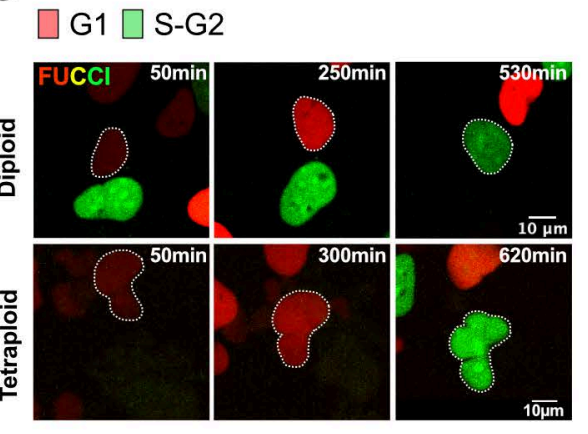

$\mathrm{H}$

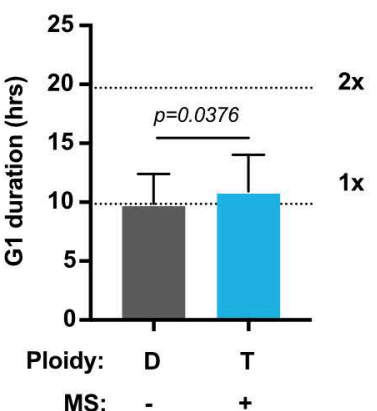

Diploid cells

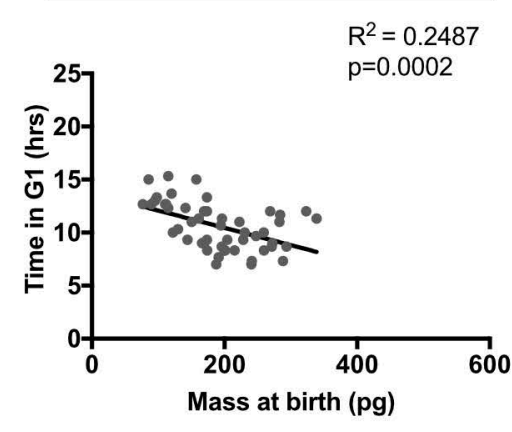

Tetraploid cells

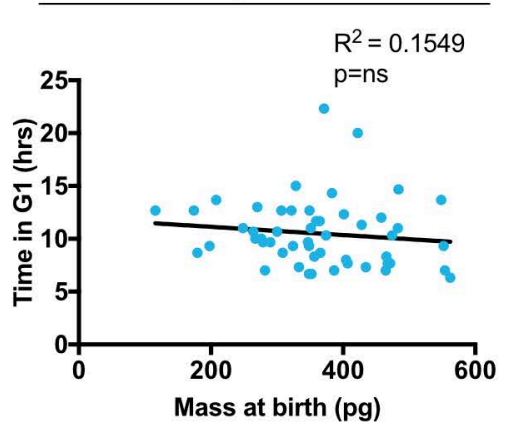

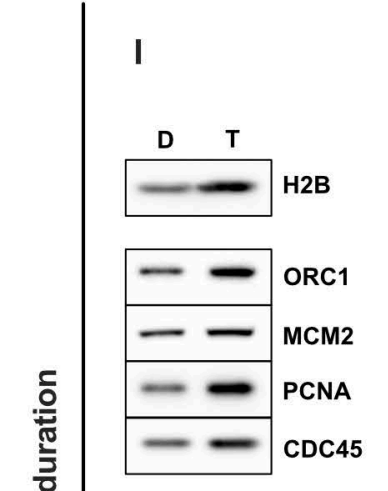

J

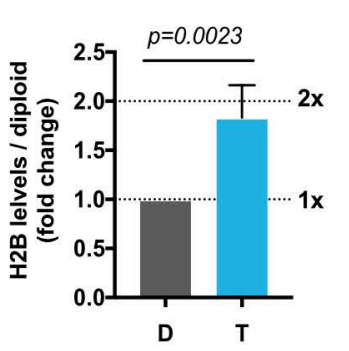

K

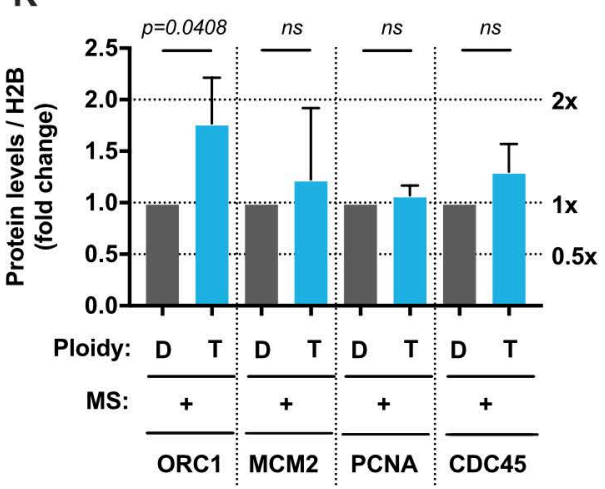

L

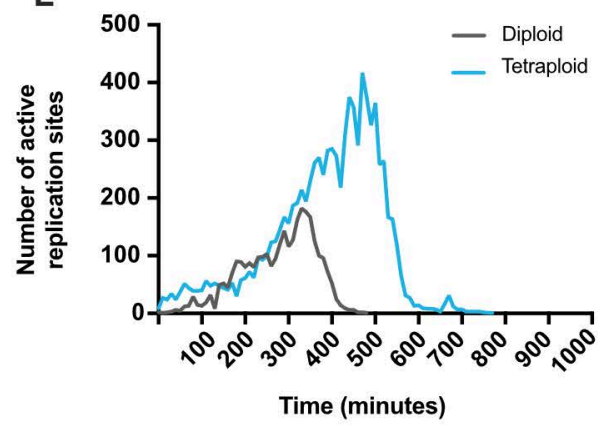

M

$p<0.0001$

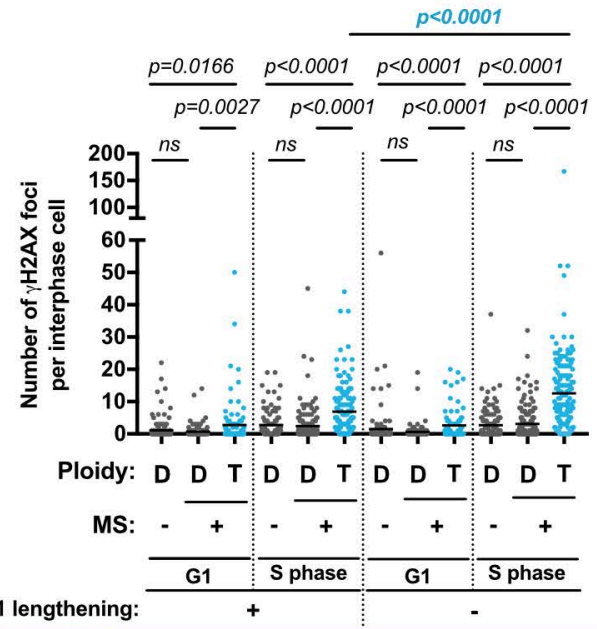


Figure 3: Lack of G1 lengthening in tetraploid cells leads to unprepared S-phase. (A) Stills of time lapse of RPE CCNB1AID FUCCI diploid and tetraploid cells. G1 cells are in red and S-G2 cells are in green. (B) Graph representing the amount of protein produced during G1 in diploid (gray) and tetraploid (blue) RPE-1 CCNB1 $1^{\text {IID }}$ FUCCI cells. (C) Schematic representation of cell sorting of $\mathrm{G} 1$ diploid and tetraploid cells. At least 50 cells were analyzed. (D) H2B, actin, ORC1, MCM2, PCNA and Cdc45 levels assessed by western blot of cell lysates obtained from diploid (left) and tetraploid (right) RPE-1 CCNB1AID FUCCI cells. The same number of cells was loaded for each condition. (E) Graph showing H2B levels normalized with diploid condition (fold change) in diploid (gray) and tetraploid (blue) cells. Mean +/- sd representing three independent experiments. (F) Graph representing the protein levels relative to $\mathrm{H} 2 \mathrm{~B}$ levels (fold change) in diploid (gray) and tetraploid (blue) cells. Mean +/- sd representing three independent experiments. (G) Left panel - Stills of time lapse of RPE FUCCI diploid and tetraploid cells. G1 cells are in red and S-G2 cells are in green. Right panel - Graph showing the time in G1 compared to the mass at birth in diploid (left panel, gray) and tetraploid (right panel, blue) RPE-1 FUCCI cells. At least 55 interphase cells from two independent experiments were analyzed. (H) Graph showing the correlation between the time in $\mathrm{G} 1$ and the mass at birth in diploid and tetraploid RPE-1 CCNB1 ${ }^{A I D}$ FUCCI cells. At least 50 interphase cells were analyzed. (I) $\mathrm{H} 2 \mathrm{~B}$, ORC1, MCM2, PCNA and Cdc45 levels assessed by western blot of cell lysates obtained from diploid (left) and tetraploid (right) RPE-1 CCNB1AID FUCCI cells with extended G1 duration. The same number of cells was loaded for each condition. (J) Graph showing H2B levels normalized with diploid condition (fold change) in diploid (gray) and tetraploid (blue) cells with extended G1 duration. Mean +/- sd representing three independent experiments. (K) Graph representing the protein levels relative to H2B levels (fold change) in diploid (gray) and tetraploid (blue) cells with extended G1 duration. Mean +/- sd representing three independent experiments. (L) Graph showing the average number of active replication sites over time in diploid (gray line) or tetraploid (blue line) RPE PCNA ${ }^{\text {chromo }}$ cells with extended G1 duration. For other representative examples, see Figure S6L. (M) Graph showing the number of $\gamma \mathrm{H} 2 \mathrm{AX}$ foci in interphase cells in diploid (gray) and tetraploid cells (blue) synchronized in G1 using 160nM (extended G1 duration) or $1 \mu \mathrm{M}$ (G1 arrest) palbociclib or released in Sphase. At least 100 interphase cells were analyzed from three independent experiments. D: Diploid. T: Tetraploid. MS: mitotic slippage. 
Fiqure 4oRxiv preprint doi: https://doi.org/10.1101/2021.07.16.452672; this version posted July 16, 2021. The copyright holder for this preprint (which was not certified by peer review) is the author/funder. All rights reserved. No reuse allowed without permission.

A

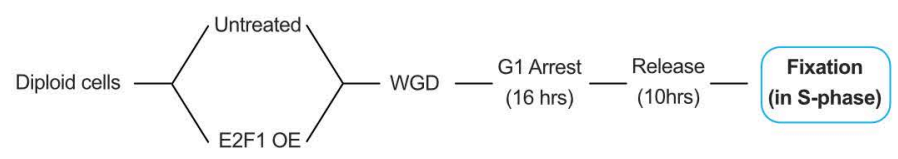

First S-phase - Diploid
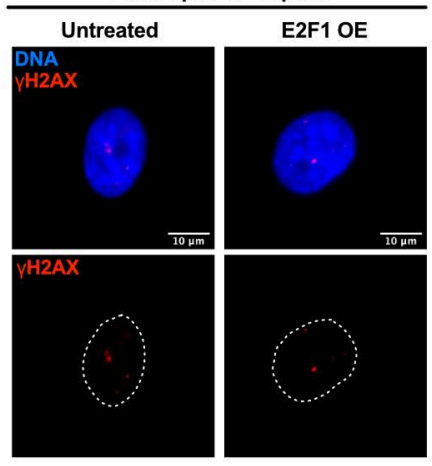

First S-phase - Tetraploid

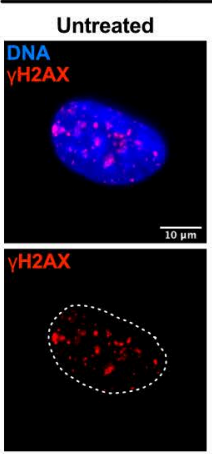

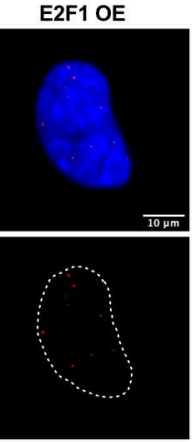

B

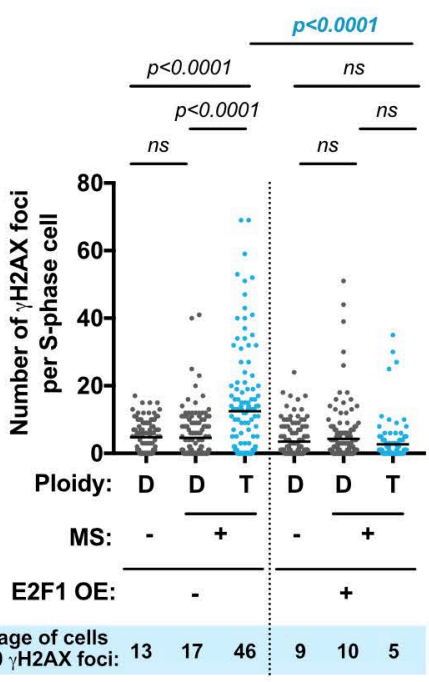

C

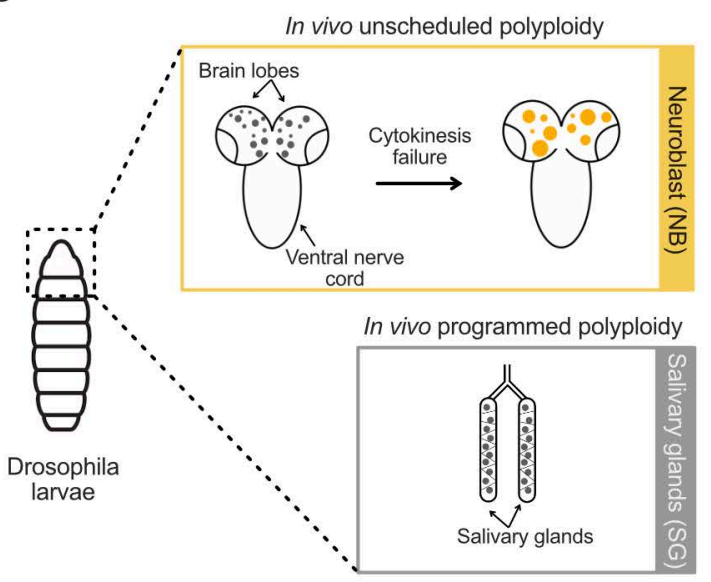

D

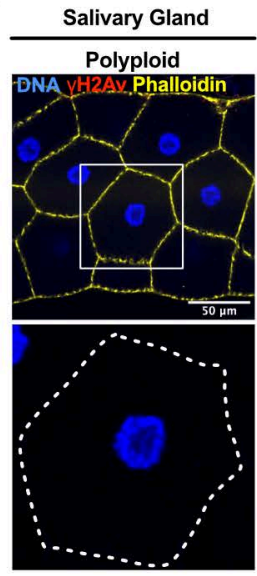

E
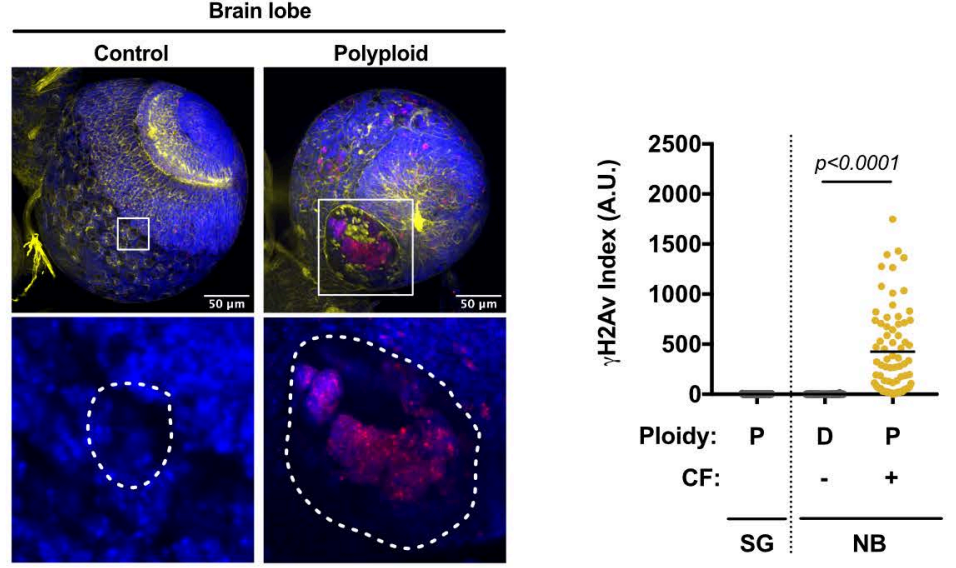

$\mathbf{F}$

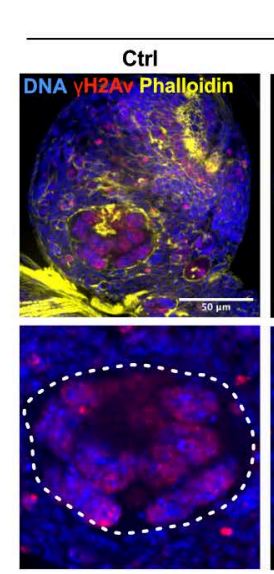

Polyploid

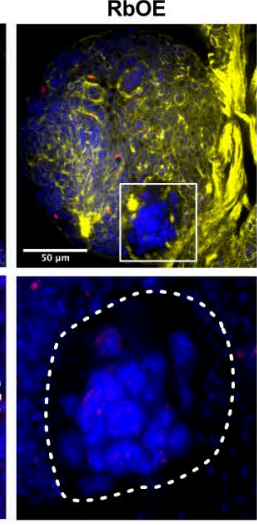

G

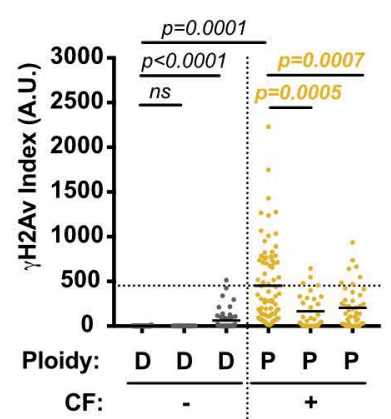

RbOE: - + - - + -

E2F1OE: - - + - - +
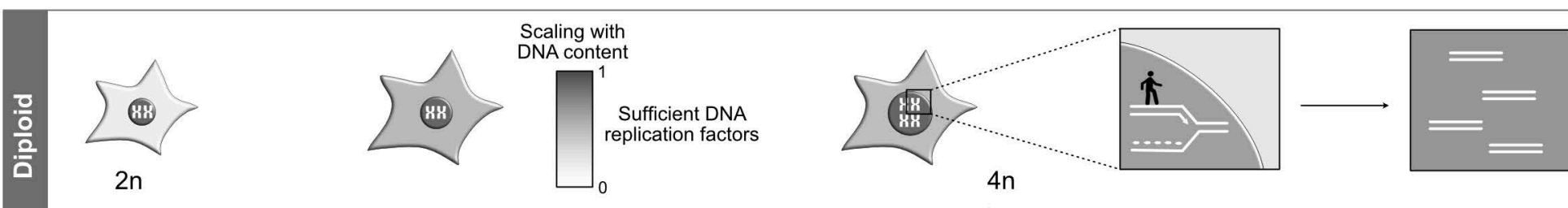

G1

S-phase
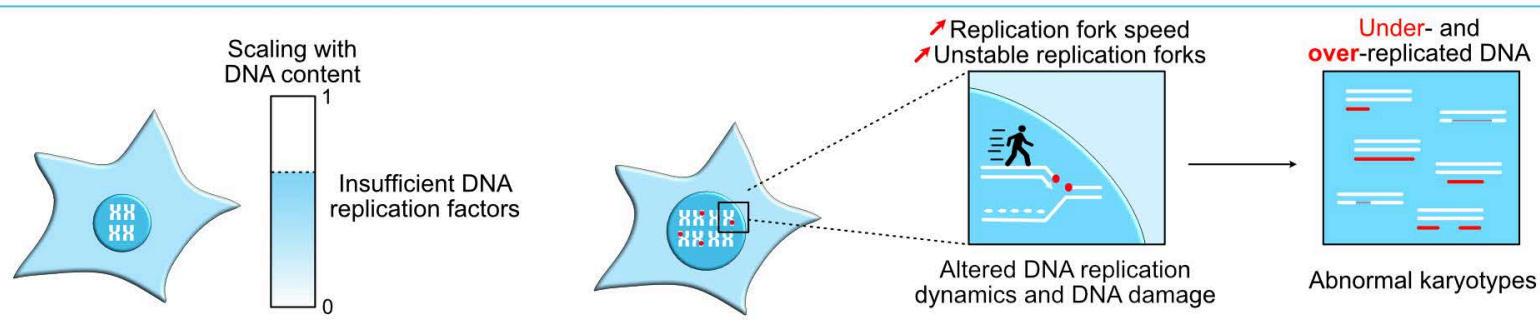

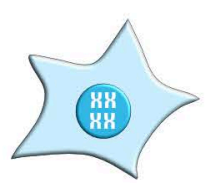

$4 n$ 
Figure 4: G1 lengthening or increased E2F1 levels are sufficient to rescue GIN in tetraploid cells and in polyploid cells in vivo.

(A) Upper panel - Schematic workflow showing the method used to overexpress E2F1. Lower panel - Representative immunofluorescence images showing DNA damage in RPE-1 tetraploid cells overexpressing or not E2F1. DNA was visualized using DAPI (in blue), DNA damage was visualized using anti- $\gamma \mathrm{H} 2 \mathrm{AX}$ antibodies (in red). (B) Graph showing the number of $\gamma \mathrm{H} 2 \mathrm{AX}$ foci per interphase cells in diploid (gray) and tetraploid (blue) RPE-1 cells released in S-phase with or without E2F1 overexpression. The percentage of interphase cells with more than $10 \gamma \mathrm{H} 2 \mathrm{AX}$ foci in diploid and tetraploid RPE-1 cells were indicated under the graph. $>100$ interphase cells were analyzed from at least three independent experiments. (C) Schematic representation of the brain of drosophila larvae. (D) Representative immunofluorescence images of drosophila brain lobe in control or sqh mutant (polyploid) and of salivary glands. DNA was visualized using DAPI (in blue), DNA damage was visualized using anti- $\gamma \mathrm{H} 2 A$ v antibodies (in red), membranes were visualized using Phalloidin (in yellow). (E) $\gamma \mathrm{H} 2 \mathrm{Av}$ index in drosophila salivary glands (SG, gray) or in diploid (gray) and polyploid (yellow) neuroblasts (NB). At least 60 cells were analyzed per condition. (F) Representative immunofluorescence images of drosophila brain lobe in control or sqh mutant (polyploid) overexpressing or not E2F1. DNA was visualized using DAPI (in blue), DNA damage was visualized using anti- $\gamma \mathrm{H} 2 \mathrm{Av}$ antibodies (in red), membranes were visualized using Phalloidin (in yellow). (G) Graph showing the $\gamma \mathrm{H} 2 \mathrm{Av}$ index in diploid (gray) and polyploid (yellow) drosophila neuroblasts overexpressing or not E2F1. At least 30 cells were analyzed per condition. (H) Tetraploid cells are not able to sense an increase in DNA content and to adapt G1 duration. In consequence, G1 duration is not scaled up with DNA content and tetraploid cells enter S-phase with an insufficient amount of replication factors generating DNA replication-dependent DNA damage and abnormal karyotypes. The dotted lines indicate the nuclear (B) or cell area ( $D$ and $F$ ). The white squares correspond to higher magnifications presented in the lower panel. D: Diploid. T: Tetraploid. P: Polyploid. CF: cytokinesis failure. MS: mitotic slippage. 